\title{
Breast cancer risk variants at 6q25 display different phenotype associations and regulate ESR1, RMND1 and CCDC170
}

\begin{abstract}
We analyzed 3,872 common genetic variants across the ESR1 locus (encoding estrogen receptor $\alpha$ ) in 118,816 subjects from three international consortia. We found evidence for at least five independent causal variants, each associated with different phenotype sets, including estrogen receptor $\left(\mathrm{ER}^{+}\right.$or $\mathrm{ER}^{-}$) and human ERBB2 (HER2+ or HER2-) tumor subtypes, mammographic density and tumor grade. The best candidate causal variants for ER- tumors lie in four separate enhancer elements, and their risk alleles reduce expression of ESR1, RMND1 and CCDC170, whereas the risk alleles of the strongest candidates for the remaining independent causal variant disrupt a silencer element and putatively increase ESR1 and RMND1 expression.
\end{abstract}

SNPs at 6q25.1 have been reported to be associated with breast cancer susceptibility in genome-wide association studies (GWAS) in women of Chinese ${ }^{1}$ and European ${ }^{2}$ ancestry. Subsequent analyses have demonstrated that SNPs in the same region are associated with breast cancer risk for $B R C A 1$ mutation carriers ${ }^{3}$ and mammographic density ${ }^{4}$, a strong breast cancer risk factor. Thus far, however, attempts to identify the candidate causal variant(s) underlying the associations have been inconclusive $^{3,5,6}$. Here we report fine-scale mapping and comprehensive analysis of the genotype-phenotype associations in this region, using dense genotyping and imputed data from the custom-designed iCOGS array, in 118,816 subjects from three consortia: the Breast Cancer Association Consortium (BCAC), the Consortium of Investigators of Modifiers of $B R C A 1$ and BRCA2 (CIMBA) and the Markers of Density Consortium (MODE). We additionally demonstrate, through functional analyses, the likely modes of action of the strongest candidate causal variants.

\section{RESULTS}

\section{Genetic epidemiological studies}

We successfully genotyped 902 SNPs across a 1-Mb region containing ESR1 in 50 case-control studies from populations of European $(89,050$ participants) and Asian (12,893 participants) ancestry in BCAC, together with 15,252 BRCA1 mutation carriers in CIMBA. Mammographic density measures were available for 6,979 women from the BCAC studies and an additional 1,621 women from the MODE Consortium, who had also been genotyped using the iCOGS array. Subsequently, the genotypes of additional variants with minor allele frequency (MAF) $>2 \%$ were imputed in all European-ancestry participants, using data from the 1000 Genomes Project as a reference. In total, data from 3,872 genotyped or imputed (imputation info score $>0.3$ ) SNPs were analyzed. Results for all SNPs associated with overall breast cancer risk $\left(P<1 \times 10^{-4}\right)$ are presented in Supplementary Table 1. Manhattan plots of the associations of these 3,872 SNPs with the main phenotypes are shown in Figure 1.

\section{Conditional analyses}

All genotyped and imputed SNPs displaying evidence of association with overall breast cancer risk in women of European ancestry
$\left(P<1 \times 10^{-4}\right)$ were initially included in forward stepwise logistic regression models for $\mathrm{ER}^{-}$and $\mathrm{ER}^{+}$breast tumor risk. The most parsimonious models (Online Methods) included four SNPs for $\mathrm{ER}^{-}$breast cancer and four SNPs for $\mathrm{ER}^{+}$breast cancer, with three SNPs being common to both models. In each model, all selected SNPs fell into a subset of five bins of correlated SNPs $\left(r^{2}>0.8\right)$. Stepwise regression models were independently fitted to breast cancer risk in the CIMBA BRCA1 mutation carriers and to mammographic density (measured as mammographic dense area; see the Online Methods for full details). For the BRCA1 mutation carriers and for mammographic dense areas, the SNPs in the best fitting models also fell within a subset of the five originally defined bins. For further analyses, we selected the directly genotyped SNP that was most significantly associated with the predominant phenotype for that bin. Regression analyses were repeated using just these five SNPs, with each representing an independent signal ${ }^{7}$. Results are presented in Table 1. Additionally, in the BCAC studies, we were able to examine SNP associations with risks of HER2 (HER2 ${ }^{+}$and HER2 ${ }^{-}$) and progesterone receptor $\left(\mathrm{PR}^{+}\right.$and $\mathrm{PR}^{-}$) tumor subtypes and with tumor grade at diagnosis. There were weak but detectable correlations between the representative SNPs for signals 1-4 (Table 1 and Supplementary Table 2). We therefore modeled the associations with each SNP conditional on the other four; these conditional risk estimates and significance levels are also presented in Table 1. At conditional significance levels of $P<1 \times 10^{-3}$, four of the lead SNPs (signals 1, 2, 4 and 5) were independently associated with risk of developing $\mathrm{ER}^{-}$breast cancer (Table 1). Another, partially overlapping, set of four SNPs (signals 1-3 and 5) was associated with $\mathrm{ER}^{+}$tumor risk (Table 2 and Supplementary Table 3), and another subset of SNPs (signals 1-4) was associated with breast cancer risk in BRCA1 mutation carriers (Table 1). The per-allele odds ratios were higher for $\mathrm{ER}^{-}$than for $\mathrm{ER}^{+}$disease for three lead SNPs (signals 1,2 and 5), whereas representative SNPs for signal 3 displayed smaller effects of similar magnitude on risk for $\mathrm{ER}^{-}$and $\mathrm{ER}^{+}$tumors. Mammographic dense area was associated with representative SNPs from signal 2 and less strongly with those from signal 1 (Table 1). We additionally carried out a meta-analysis of the SNP associations

A full list of authors and affiliations appears at the end of the paper. 
a
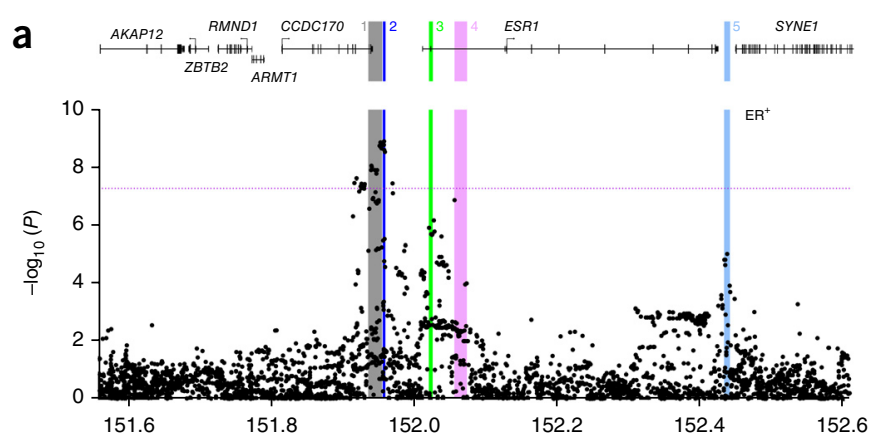

b
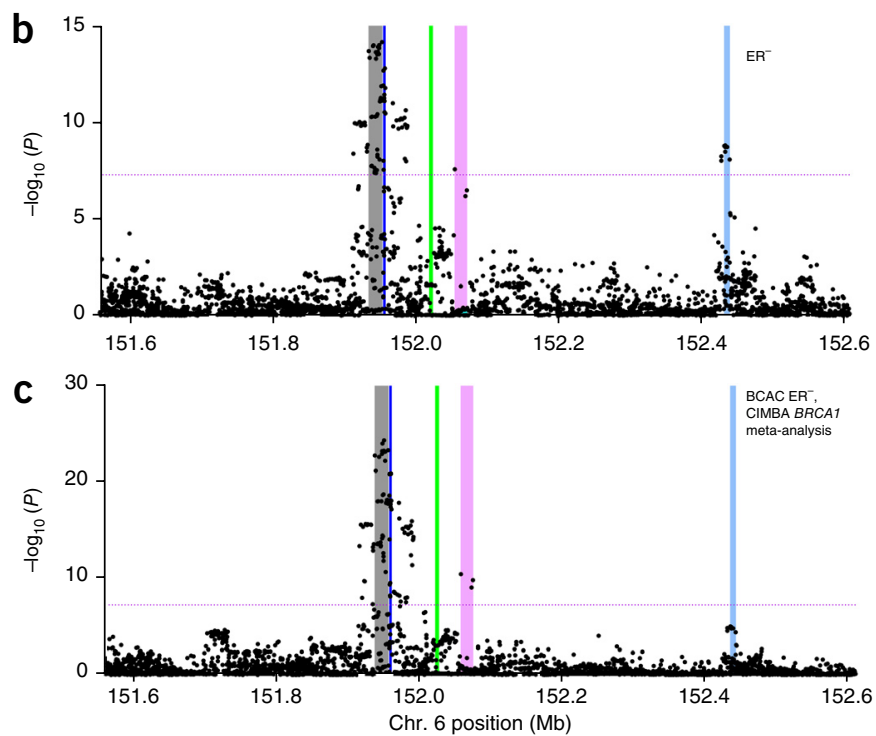

d
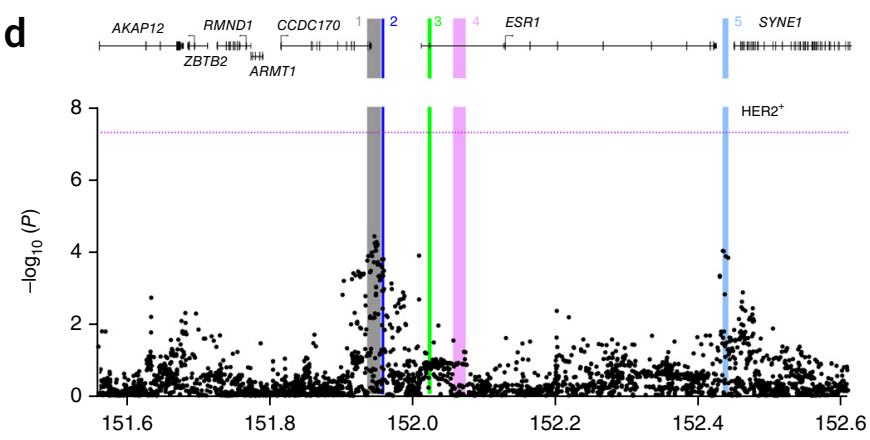

e
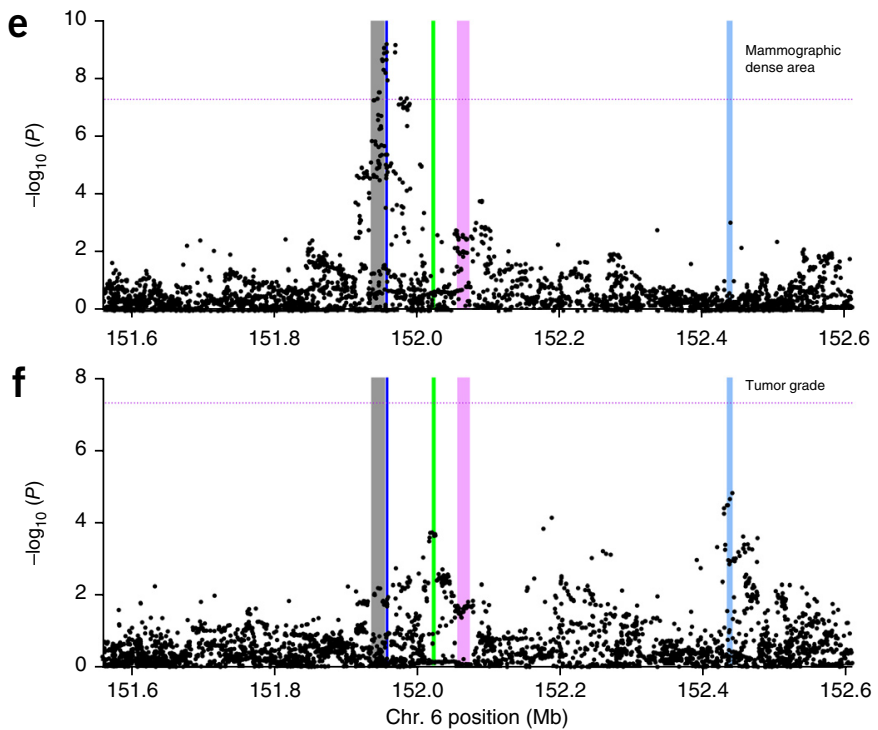

Figure 1 Association results for all SNPs with six phenotypes. (a-f) The phenotypes analyzed include risk of ER+ breast cancer in BCAC (a), risk of $\mathrm{ER}^{-}$breast cancer in BCAC (b), risk of triple-negative breast cancer, derived from the CIMBA meta-analysis of BRCA1 mutation carriers with ER- tumors (c), risk of HER2+ breast cancer in BCAC (d), mammographic dense area in MODE (e) and tumor grade after adjustment for ER status in BCAC (f). $P$ values for each SNP (from unconditional logistic regression) are shown plotted as the negative log-transformed $P$ value against relative position across the locus. A schematic of the gene structures is shown above a and $\mathbf{d}$. The physical positions of signals $1-5$ are shown as colored, numbered stripes. Dotted horizontal lines indicate the genome-wide significance level.

with breast cancer risk for CIMBA BRCA1 mutation carriers and risk of $\mathrm{ER}^{-}$tumors in BCAC. We anticipated that this analysis would increase statistical power to detect $\mathrm{ER}^{-}$risk signals, and, indeed, it did strengthen the evidence for association of SNPs representing signals 1-4 but not signal 5 , which showed no association with breast cancer risk in BRCA 1 mutation carriers (Table 1).

\section{Tumor subtype and grade analyses}

We next explored the associations of each signal with specific tumor subtype combinations and with tumor grade (Fig. 1f, Table 2 and Supplementary Tables 3-5). The representative SNPs at two signals ( 3 and 5) were strongly associated with high-grade disease, after adjusting for ER status $\left(P<1 \times 10^{-3}\right.$; Table 2 (bottom line) and Supplementary Table 5). Among $\mathrm{ER}^{-}$tumors, three signals (1,2 and 4) were associated with triple-negative $\left(\mathrm{ER}^{-} \mathrm{PR}^{-} \mathrm{HER} 2^{-}\right)$and high-grade tumors, as well as the rarer $\mathrm{ER}^{-} \mathrm{PR}^{-} \mathrm{HER} 2^{+}$subtype, with similar odds ratios (Table 2 and Supplementary Tables 3 and 5). However, signal 5 was more strongly associated with $\mathrm{ER}^{-} \mathrm{PR}^{-} \mathrm{HER}{ }^{+}$disease (odds ratio $(\mathrm{OR})=1.24$, $95 \%$ confidence interval $(\mathrm{CI})=1.12-1.37 ; P=2.4 \times 10^{-5}$; Table 2$)$ than with the triple-negative subtype $(\mathrm{OR}=1.08,95 \% \mathrm{CI}=1.01-1.15$; $P=0.016$; Table 2, case-only $P=0.021$; Supplementary Table 5), consistent with the lack of association for breast cancer in BRCA1 mutation carriers, in whom tumors are predominantly triple negative ${ }^{8}$.

\section{Haplotype analysis}

We next explored the combined effects of the same five signalrepresentative genotyped SNPs (Supplementary Table 6). Haplotypespecific effects were consistent with additive effects of the individual signal-representative SNPs. In particular, haplotype 22221 (all minor alleles except for signal 5 ; frequency $=0.005$ ) was associated with the largest increased risks of both $\mathrm{ER}^{+}(\mathrm{OR}=1.38,95 \% \mathrm{CI}=1.11-1.71$; $\left.P=3.3 \times 10^{-3}\right)$ and $\mathrm{ER}^{-}\left(\mathrm{OR}=2.34,95 \% \mathrm{CI}=1.76-3.10 ; P=3.5 \times 10^{-9}\right)$ tumors; this group includes the triple-negative $\left(\mathrm{ER}^{-} \mathrm{PR}^{-} \mathrm{HER} 2^{-}\right)$ tumor subtype (detected via the meta-analysis of BCAC subjects with $\mathrm{ER}^{-}$tumors and CIMBA BRCA1 mutation carriers; $P=8 \times 10^{-10}$ ). Haplotype 22111 (frequency $=0.02$ ) was associated with the highest risk of HER2 $2^{+}$tumors $\left(\mathrm{OR}=1.5,95 \% \mathrm{CI}=1.21-1.87 ; P=3 \times 10^{-4}\right)$ and with mammographic dense area $(\beta$ coefficient $=0.45,95 \% \mathrm{CI}=$ 0.20 to $0.69 ; P=3 \times 10^{-4}$ ).

\section{Associations in Asian-ancestry studies}

We examined the associations of the five signal-representative SNPs in the nine Asian-ancestry studies in BCAC (Supplementary Table 7). All five displayed allelic associations in the same direction as in Europeans, with overlapping confidence intervals, consistent with the hypothesis that the same candidate causal variants determine risk in both populations. 


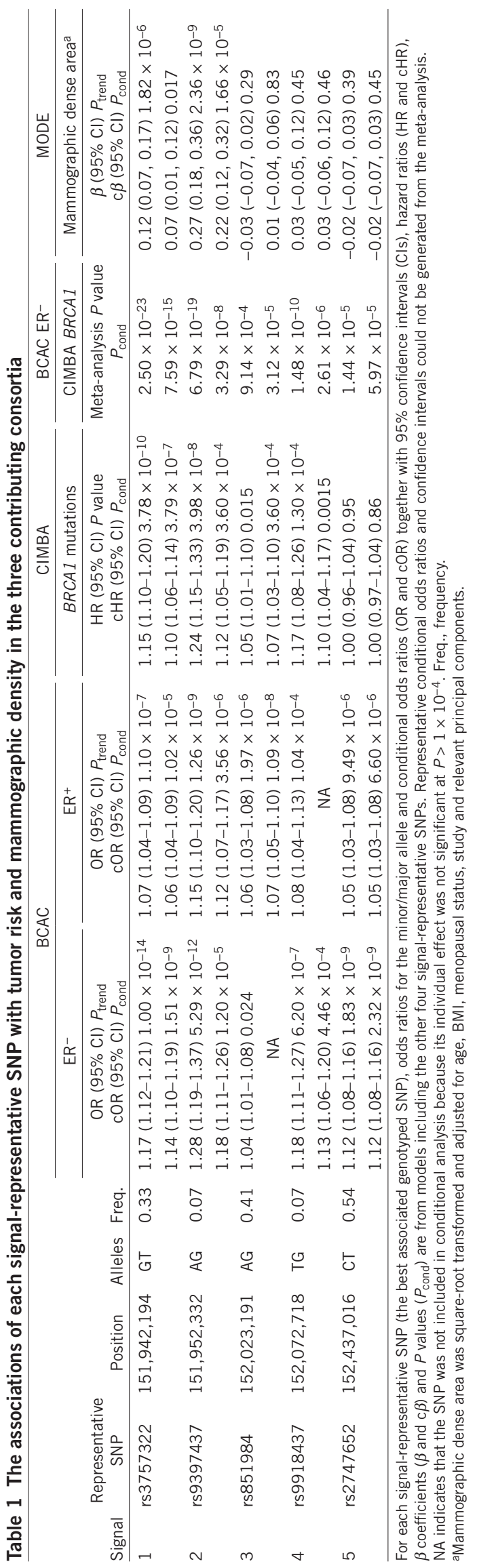

\section{Determining the candidate SNPs within each signal}

To identify the potential causal variants to be taken forward for functional analysis, we determined the most significant SNP association within each signal and then calculated the likelihood ratio of every other SNP relative to that SNP. We assumed that SNPs with a likelihood of $<1: 100$ (ref. 9) in comparison with the most significant SNP for each signal could be excluded from consideration as potentially causative variants. On the basis of the assumption that, within a given signal, the same variant(s) would be driving all observed phenotype associations, we derived the list of most likely causal SNPs for each signal. We used the results from one of two analyses to define the list of potentially causal SNPs for each signal: the meta-analysis of BCAC subjects with $\mathrm{ER}^{-}$disease and CIMBA BRCA1 mutation carriers for signals 1,2 and 4 , which were most strongly associated in this analysis, and overall breast cancer risk in BCAC for signals 3 and 5 . These lists of unexcluded variants are presented in Table 3 and are highlighted in Supplementary Table 1.

In signal 1, the most strongly associated variant was rs2046210 (the original Asian GWAS hit ${ }^{1,10}$ ), with nine other variants (likelihood ratios <100:1, $r^{2} \geq 0.89$ with rs2046210; spanning $151,935,539-151,954,127)$ remaining as strong causal candidates. In signal 2, the best causal candidate was SNP rs12173570, with two other candidates remaining (likelihood ratios $<100: 1, r^{2} \geq 0.75$ with rs12173570; spanning 151,955,914-151,958,815). The European GWAS SNP, rs3757318l (ref. 2), is most strongly correlated with rs12173570 $\left(r^{2}>0.45\right)$. In signal 3 , the best causal candidate was rs851984, with three other candidates remaining (likelihood ratios $<100: 1, r^{2}=0.99$; in two ESR1 introns spanning 152,020,390$152,024,985)$. In signal 4 , the top candidate was rs9918437, and two other candidates spanned another segment of an ESR1 intron at 152,055,978-152,072,718 (approximately $30 \mathrm{~kb}$ telomeric of signal 3 ; likelihood ratios $<100: 1, r^{2}>0.81$ with rs9918437). In signal 5, the strongest candidate causal SNP was rs2747652 (also the representative SNP for signal 5 in Table 1), and there were five other candidates (likelihood ratios <100:1, $r^{2}>0.97$ with rs2747652; spanning $152,432,902-152,440,522)$ in the intergenic region between ESR1 and SYNE1. Across the five signals, we were able to exclude all but 26 of the original 3,872 variants from being potentially causal.

\section{Local gene expression analyses}

We used four techniques to assess associations between candidate causal variants (or available proxy SNPs) in the five signals and local gene expression. (i) ER protein expression, measured by immunohistochemistry in normal breast tissue samples from 150 postmenopausal donors, identified a significant correlation of the risk alleles of signal 1 SNPs with reduced ER levels (Fig. 2a and Supplementary Figs. 1 and 2). (ii) ESR1 expression in breast tumors and adjacent normal breast tissue from the Molecular Taxonomy of Breast Cancer International Consortium (METABRIC) study was compared relative to signalrepresentative SNP allele (Fig. 2 b and Supplementary Table 8). In patients with $\mathrm{ER}^{-}$tumors, risk allele carriers had lower median ESR1 expression in normal, tumor-adjacent tissue than homozygotes for the protective allele at signals 1,4 and 5 , although none of the differences were statistically significant. By contrast, in patients with $\mathrm{ER}^{+}$tumors, risk allele carriers had higher median ESR1 expression in normal, tumor-adjacent tissue than homozygotes for the protective allele at signals 1, 3 and 5. (iii) Allele-specific expression (ASE) analysis, using RNA sequencing (RNA-seq) data from breast tumor samples and SNP array genotype data from The Cancer Genome Atlas (TCGA) ${ }^{11}$, showed allelic imbalances in ESR1 expression among heterozygotes for proxy SNPs in signals 1-3 (Fig. 2c and Supplementary Table 9). 


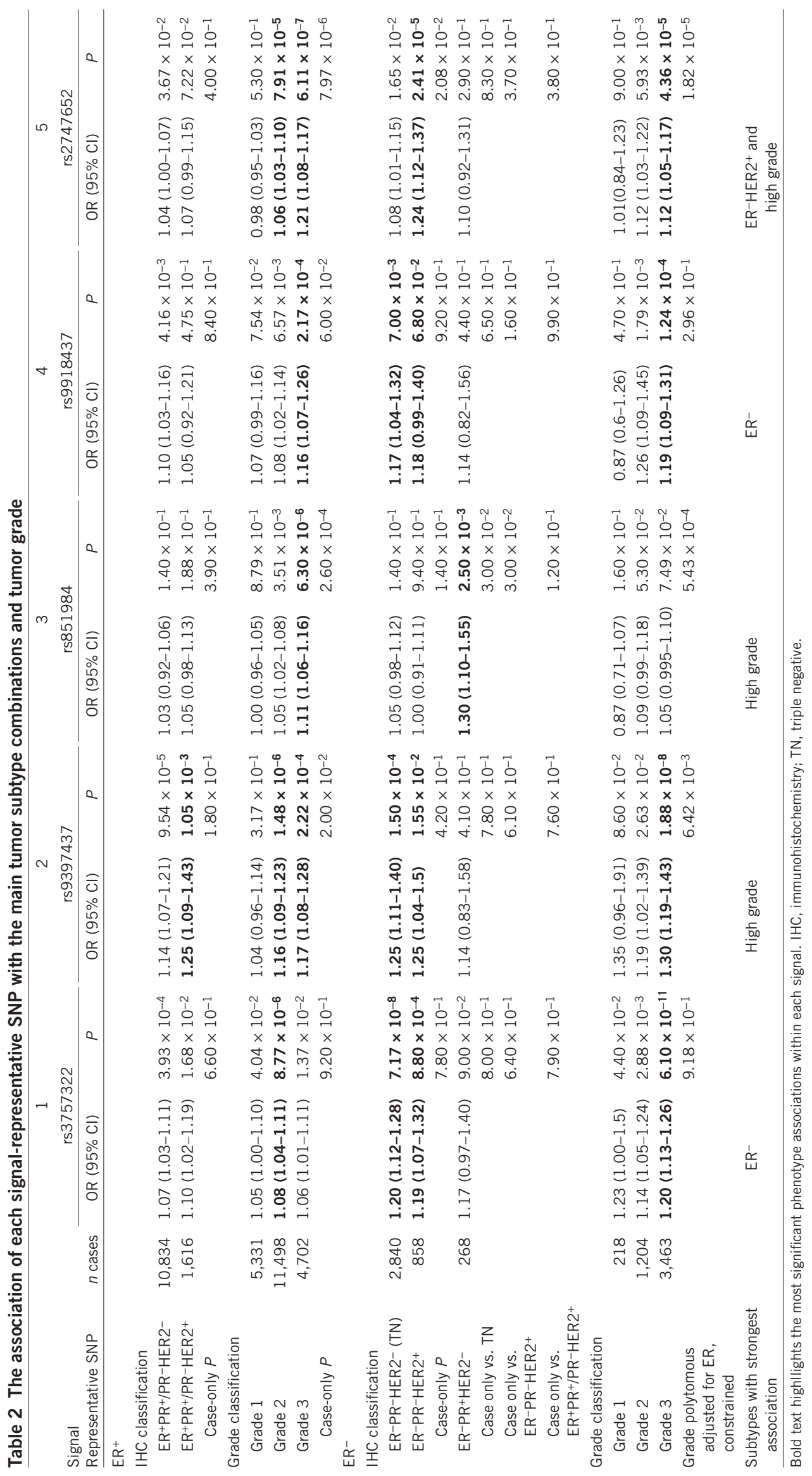




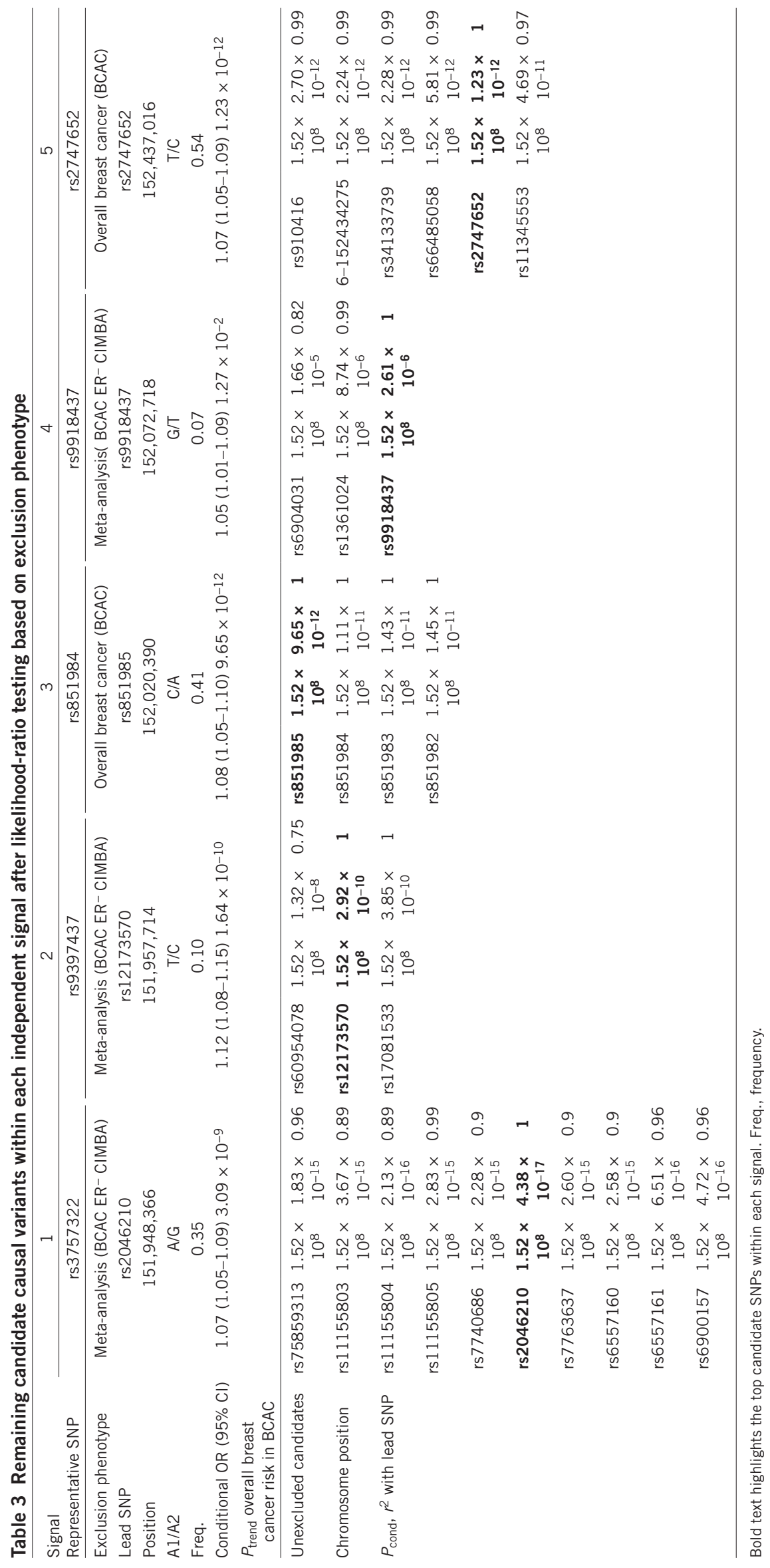


a

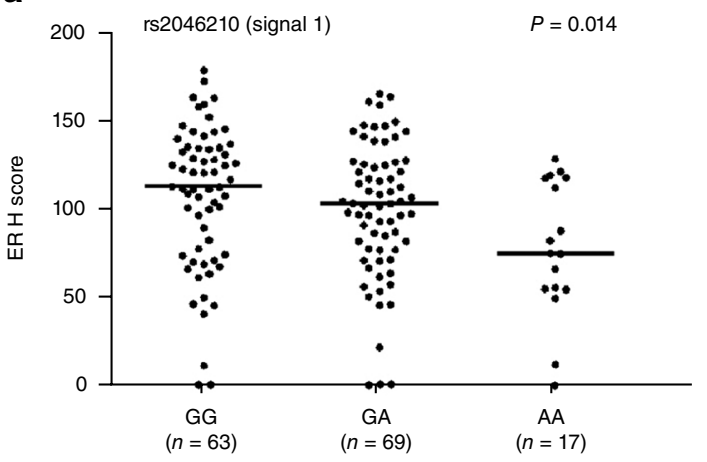

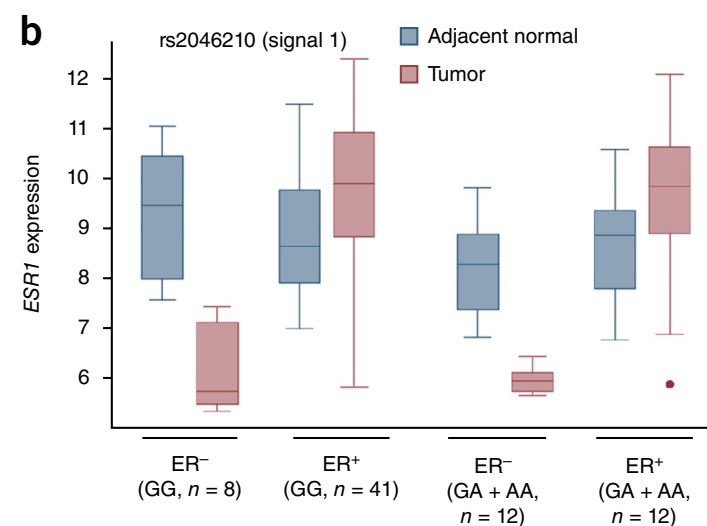
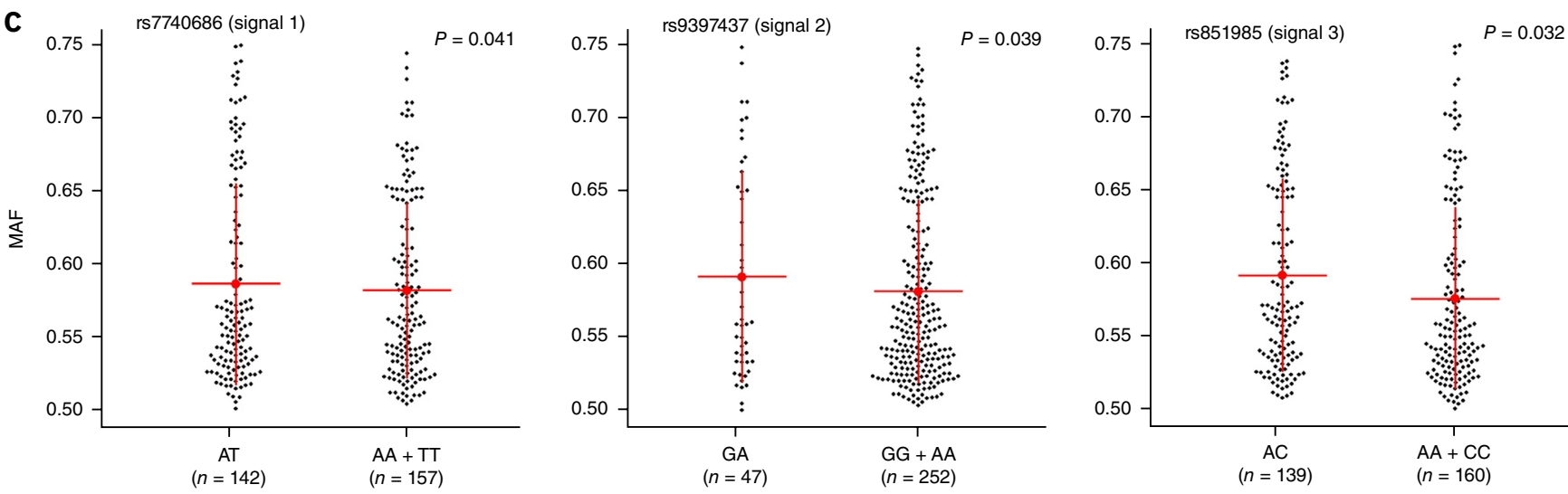

Figure 2 ER expression and allelic imbalance correlate with signal 1 SNPs. (a) Negative correlation between the signal 1 SNP rs2046210 and ER protein expression. Black dots represent ER expression from individual samples measured by immunohistochemistry (H score). Horizontal lines represent the mean $\mathrm{H}$ score for each genotype. The $P$ value was calculated using a Spearman rank correlation test. (b) Box plots of ESR 1 gene expression ( $\log _{2}$ transformed) in breast tumor and adjacent normal samples. Boxes extend from the 25th to the 75th percentile, horizontal bars represent the median, whiskers indicate the full range of ESRI expression and outliers are represented as circles. (c) Allelic imbalance in ESR 1 expression by genotypic status at breast cancer risk variants. Data are classified according to the genotypes at risk SNPs (heterozygous versus homozygous). Black dots represent the average major allele fraction of the marker SNPS across ESR 1 for an individual from TCGA with breast cancer. Red lines and whiskers correspond to means \pm 1 s.d. For rs7740686 (signal 1) and rs9397437 (signal 2), Levene's test (equality of variances) was used to calculate the $P$ values; for rs 851985 (signal 3), a two-tailed $t$ test (equality of means) was used to calculate the $P$ value.

Similar imbalances in CCDC170 expression were detected among heterozygotes for signal 2 SNP rs9397437 and in RMND1 expression with signal 3 SNP rs851983 (Supplementary Table 9). Such allelic imbalances indicate that risk alleles at these signals are associated with expression differences in local genes, but they do not indicate the directions of association. (iv) Expression quantitative trait locus (eQTL) analysis using the Gene-Tissue Expression (GTEx) database identified a significant association for SNPs in signal 3 with CCDC170 expression in normal breast tissues (Supplementary Table 10). We also performed cis-eQTL analyses on the 12 flanking genes in 135 normal breast tissue samples from the METABRIC study; however, no additional associations were detected (Supplementary Table 11).

\section{Bioinformatic and chromatin analyses}

Analysis of cis enhancer-gene interactions using PreSTIGE ${ }^{12}$ showed evidence of multiple regulatory elements coinciding with signals 1-3 in ER ${ }^{+}$MCF-7 breast cancer cells (Fig. 3a and Supplementary Fig. 3). A 'super-enhancer', associated with high levels of acetylation of histone $\mathrm{H} 3$ at lysine 27 (H3K27ac), was also identified in MCF-7 cells and encompasses the top risk-associated SNPs in these three signals (Fig. 3a and Supplementary Fig. 3) ${ }^{13}$. This super-enhancer was most readily detectable in MCF-7 cells and was not observed in other breast cancer cell lines, normal mammary epithelial cells or other tissues analyzed (Supplementary Fig. 4). Chromatin conformation capture (3C) experiments demonstrated that elements within signals 1 and 2 physically interacted with the promoters of ESR1, RMND1-ARMT1 and CCDC170 in MCF-7 and T-47D cells (Fig. 3b and Supplementary Fig. 5a,b). Furthermore, we detected interactions between signals 3-5 and ESR1 and/or RMND1-ARMT1 promoters (Fig. 3c,d and Supplementary Fig. 5c,d). The majority of these interactions were restricted to MCF-7 and T-47D cells (ER ${ }^{+}$breast cancer cell lines), but the RMND1-ARMT1 interactions were also detected in either Bre-80 or MCF10A cells (ER- 'normal' breast cell lines; Fig. 3b-d and Supplementary Fig. 5b-d). The 3C-identified interactions for each signal are summarized in Supplementary Table 12.

\section{Prioritizing candidate SNPs for functional assays}

We applied a combination of in silico and in vitro analyses to prioritize candidate causal SNPs for functional follow-up, using previous observations that common cancer susceptibility alleles are enriched in cis-regulatory elements ${ }^{14-16}$. First, Table 3 showed that 19 of the 26 top candidates overlapped DNase I-sensitive sites and were associated with enhancer-enriched histone marks such as dimethylation of histone $\mathrm{H} 3$ at lysine 4 (H3K4me2) and $\mathrm{H} 3 \mathrm{~K} 27 \mathrm{ac}$ in MCF-7 and HMEC breast cells, indicative of putative regulatory elements (PREs) (Supplementary Fig. 6). In electromobility 
Figure 3 Chromatin interactions across the $6 q 25.1$ risk region. (a) Signals $1-5$ are numbered and shown as colored stripes. RMND1, ARMT1, CCDC170 and ESR1 gene structures are depicted with exons (vertical bars) joined by introns (lines). Gene-enhancer predictions from PreSTIGE ${ }^{12}$, ChIP-seq binding profiles for H3K27ac ${ }^{13}$ and Encyclopedia of DNA Elements (ENCODE) RNA polymerase II ChIA-PET interactions in MCF-7 cells are shown. (b-d) $3 \mathrm{C}$ anchor points (3C baits) and interrogated sequences ( $3 \mathrm{C}$ regions) are depicted as black boxes and gray shading, respectively. $3 \mathrm{C}$ interaction profiles in $\mathrm{ER}^{+} \mathrm{MCF}-7$ and ER $^{-}$Bre- 80 breast cell lines are shown for signals 1 and 2 (b), signals 3 and 4 (c) and signal 5 (d). 3C libraries were generated with EcoRI, with the anchor point set at the ESR1, RMND1-ARMT1 or CCDC170 promoter region. Graphs present the results from three biological replicates; error bars, s.d.

shift assays (EMSAs), 11 of these 19 SNPs altered the binding affinity of transcription factors in vitro (Supplementary Fig. 7). Of these, seven fell within promoter-specific long-range interactions identified by $3 \mathrm{C}$ (Fig. 3 and Supplementary Fig. 5). The 7 SNPs prioritized for further detailed analyses included 2 of 10 remaining candidates in signal 1 (rs7763637 and rs6557160), 1 of 3 candidates in signal 2 (rs17081533), 2 of 4 candidates in signal 3 (rs851982 and rs851983), 1 of 3 candidates in signal 4 (rs1361024) and 1 of 6 candidates in signal 5 (rs910416) (Supplementary Table 12).

\section{Luciferase reporter assays}

The regulatory capabilities of the PREs overlapping each signal and the effects of the seven prioritized candidate SNPs were examined in luciferase reporter assays in the $\mathrm{ER}^{+} \mathrm{MCF}-7$ and BT-474 and the ER ${ }^{-}$Bre-80 breast cell lines. PRE constructs containing the reference alleles of the prioritized SNPs for signals 1, 2, 4 and 5 significantly increased associated target gene promoter activity when cloned in either direction, indicating that they act as orientation-independent transcriptional enhancers. In contrast, a PRE containing the reference alleles of the signal 3 candidates ablated target gene promoter activity, but only when cloned in the forward direction, suggesting that this region acts as an orientation-dependent silencer (Fig. 4 and Supplementary Figs. 8-10). Notably, inclusion of the minor (risk) alleles of individual candidate SNPs in signals 1, 2 and 5 (rs6557160, rs17081533 and rs910416) significantly reduced ESR1 and RMND1 promoter activity but had no effect on the $A R M T 1$ or $C C D C 170$ promoters. However, inclusion of the signal 1 haplotype significantly decreased ESR1, RMND1 and CCDC170 promoter activity (Fig. 4 and Supplementary Figs. 8 and 9). Inclusion of the individual minor (risk) allele of signal 4 SNP rs1361024 or signal 3 SNP rs851983 in the respective constructs had no additional effects. In contrast, inclusion of the signal 3 minor (risk) allele of rs 851982 or the haplotype construct increased ESR1 promoter activity in ER ${ }^{+}$MCF-7 and BT-474 cells and RMND1 promoter activity

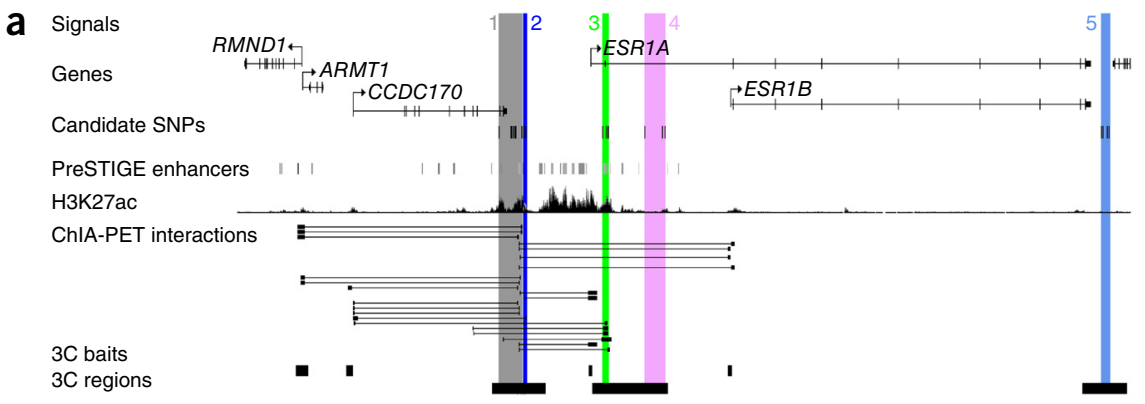

b
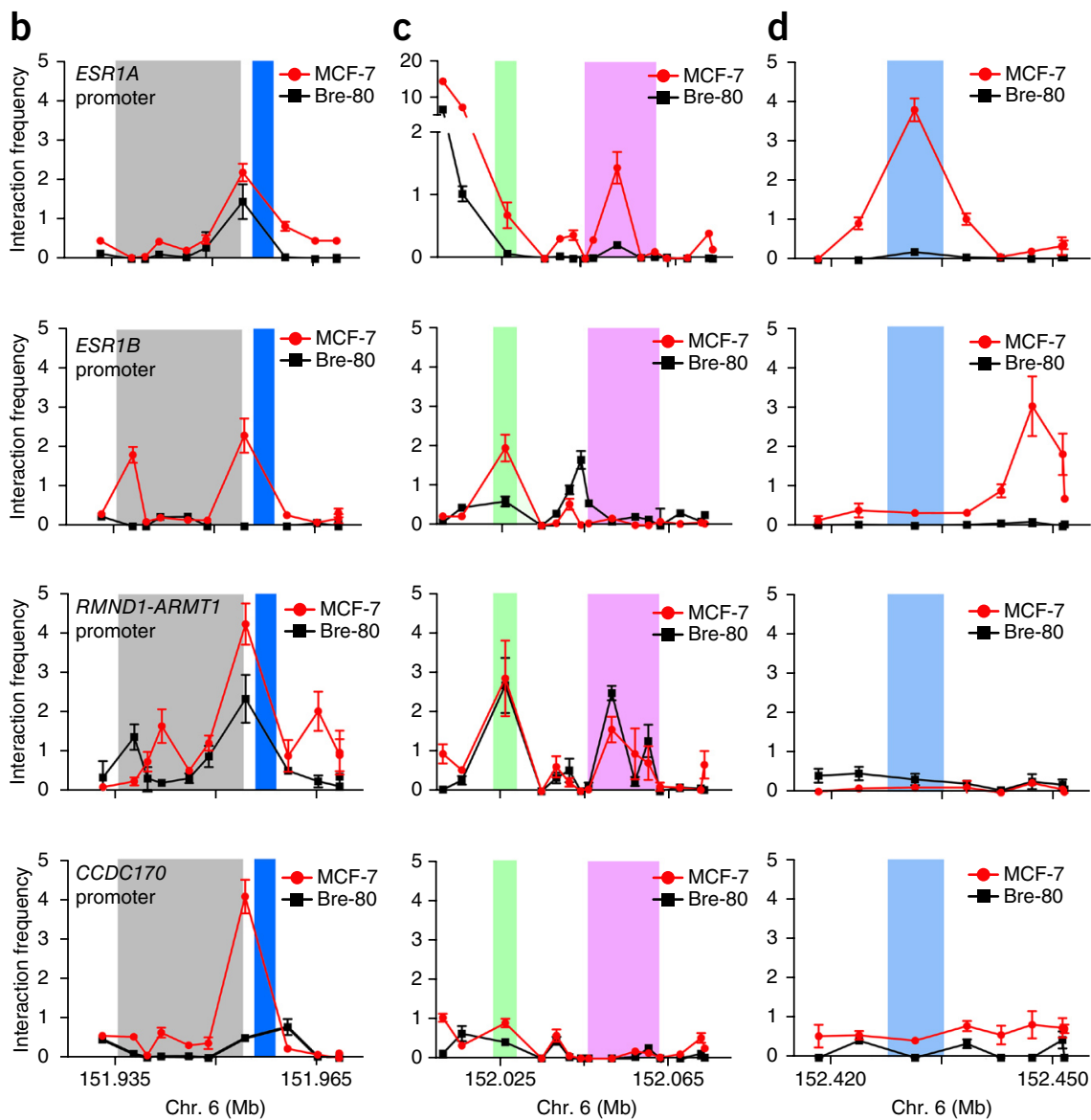

in all three cell lines (Fig. 4, Supplementary Figs. 8 and 9, and Supplementary Table 12).

\section{Transcription factor binding analyses}

We used both bioinformatic analyses and functional studies to examine DNA-protein interactions for the seven prioritized SNPs. In silico prediction tools including intragenomic replicates (IGR) ${ }^{17}$, HaploReg ${ }^{18}$ and Alibaba2 (ref. 19) predicted that all seven SNPs alter transcription factor binding (Supplementary Fig. 11 and Supplementary Table 13).

Competition with known transcription factor binding sites suggested the identity of bound proteins for four of the prioritized SNPs, including GATA3 binding to the minor (risk) allele of signal 3 SNP rs851982 and CTCF binding to the minor allele of a second signal 3 candidate, rs851983, as well as the common (protective) allele of signal 4 candidate rs1361024 and MYC binding to the common allele of signal 5 candidate rs910416 (Supplementary Fig. 12 and Supplementary Table 12). Additional well-established breast cell transcription factors, such as ER itself and FOXA1, were also assessed but did not display 
Figure 4 Risk alleles reduce ESR 1 and RMND1 promoter activity. Luciferase reporter assays were performed following transient transfection of ER+ MCF-7 breast cancer cells. PREs containing the major SNP alleles were cloned downstream of target gene promoter-driven luciferase constructs (prom) for the creation of reference (Ref-PRE) constructs. Minor SNP alleles were engineered into the constructs and are designated by the rsID of the corresponding SNP. "Haplotype" denotes a construct that contains the minor alleles of both candidate SNPs within signal 1 or 3 . Error bars, $95 \%$ confidence intervals from three independent experiments. $P$ values were determined by two-way ANOVA followed by Dunnett's multiplecomparisons test: ${ }^{* *} P<0.01,{ }^{* *} P<0.001$.

competitive binding to any prioritized SNP sites (Supplementary Fig. 13). Chromatin immunoprecipitation (ChIP) confirmed enrichment of GATA3 binding to DNA overlapping signal 3 candidate rs851982, but no difference between the alleles, and confirmed CTCF binding to the region overlapping signal 4 candidate rs 1361024 in BT-474 cells (Fig. 5a and Supplementary Fig. 14).

CTCF also bound to the region encompassing signal 3 candidate rs 851983 (Fig. 5a, Supplementary Fig. 14 and Supplementary Table 12). CTCF mediates long-range chromatin looping; therefore, to assess the potential impact of signal 4 candidate rs 1361024 and signal 3 candidate rs851983 on chromatin interactions, we performed allelespecific 3C in heterozygous cell lines. Sequence profiles indicated that the protective $\mathrm{G}$ allele of signal 4 candidate rs 1361024 increases looping between this enhancer and the ESR1 and RMND1 promoters (Fig. 5b and Supplementary Fig. 15a). We found no evidence for allele-specific looping between the silencer overlapping signal 3 and local gene promoters (Supplementary Fig. 15b).

\section{DISCUSSION}

The fine-scale mapping, bioinformatic and functional analyses presented here provide evidence for the existence of at least five different genetic variants, each with a direct effect on breast cancer risk in Europeans, findings also supported by the limited available data in Asian populations. These variants are distributed upstream, within introns and downstream of ESR1, each in a region, which we have demonstrated via reporter assays, is regulatory for ESR1. Some may additionally regulate other local genes, such as RMND1, ARMT1 and $C C D C 170$, previously reported to be co-regulated with ESR1 (ref. 20). Of note, the four sites more strongly associated with risks of $\mathrm{ER}^{-}$than $\mathrm{ER}^{+}$tumors (signals 1, 2, 4 and 5) all overlap enhancer regions, and our evidence indicates that the minor (risk) alleles of candidate causal variants, within each of these enhancers, act to reduce expression of ESR1, RMND1 and CCDC170. In contrast, signal 3-which is associated with smaller but equal risks of developing both $\mathrm{ER}^{-}$and $\mathrm{ER}^{+}$tumors-overlaps a putative gene silencer, and the risk alleles of the candidate causal variants here increase ESR1 and RMND1 expression. Furthermore, we have demonstrated altered binding of looping factor CTCF to candidate causal SNPs in signals 3 and 4, with evidence that the risk allele of signal 4 candidate rs1361024 abrogates binding and reduces chromatin looping between this enhancer element and the promoters of ESR1 and RMND1. We also provided evidence that signal 5 candidate rs 910416 may display allele-specific binding of MYC.

Notably, the previously unrecognized signal 5 candidates, downstream of ESR1, significantly increase the risk of developing $\mathrm{ER}^{-} \mathrm{PR}^{-} \mathrm{HER} 2^{+}$tumors (a specific subtype shown to be more responsive to the drug trastuzumab) in contrast to the triplenegative (ER-PR ${ }^{-} \mathrm{HER}^{-}$) tumor subtype, which has already been reported to be associated with other signals at $6 \mathrm{q} 25$ as well as 19 p13 (ref. 21) and 5p15 (TERT) 22 . We also found evidence that the candidate causal variants at signals 3 and 5 predispose to aggressive, high-grade breast cancer, independently of ER status.
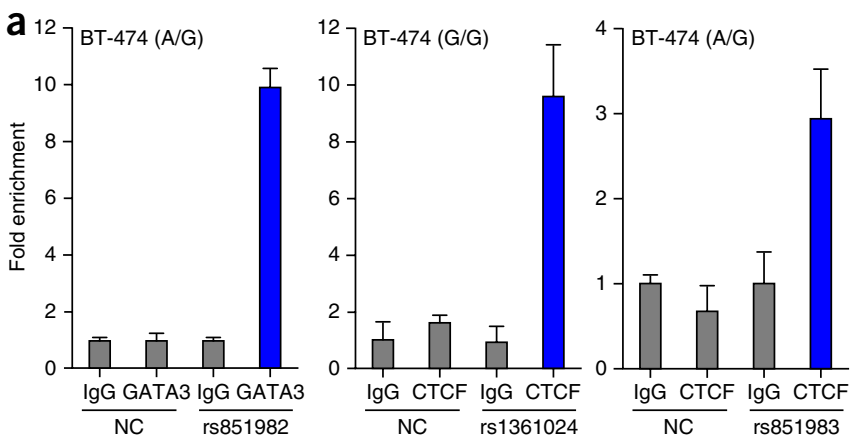

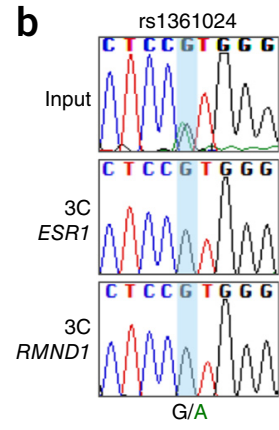

Figure 5 GATA3 and CTCF binding in vivo.

(a) ChIP and quantitative PCR (qPCR) assays using

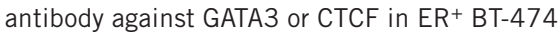
breast cancer cells. A region within the second intron of ESR1 served as a negative control (NC). Normal rabbit IgG was used as a non-specific antibody control. Graphs present the results of two biological replicates; error bars, s.d. (b) $3 C$ followed by sequencing for the signal 4 PRE containing rs1361024 in heterozygous ER+ MCF-7 breast cancer cells shows allele-specific chromatin looping. The chromatograms represent one of three independent $3 \mathrm{C}$ libraries generated and sequenced. 
Mammographic density adjusted for age and body mass index (BMI), which describes the variation in epithelial and stromal tissue on a mammogram, is one of the strongest known risk factors for breast cancer ${ }^{23}$ and has been shown to have a shared genetic basis with breast cancer, mediated through a large number of common variants ${ }^{24}$. Associations between ESR1 SNPs and mammographic density have previously been reported ${ }^{25-27}$, but, in this detailed analysis, only signal 2 was significantly associated with mammographic dense area $\left(P=1.7 \times 10^{-5}\right)$, although signal 1 also showed some evidence of an effect in the conditional analysis $(P=0.017)$. Although adjusting the breast cancer analysis of signal 2 for mammographic dense area produced some attenuation of the associated effect, the lead SNP remained significantly associated with breast cancer risk (unconditional $\mathrm{OR}=1.30,95 \% \mathrm{CI}=1.13-1.49 ; P=0.00024 ;$ OR conditional on dense area $=1.24,95 \% \mathrm{CI}=1.08-1.43 ; P=0.0025)$, suggesting either that the mechanism by which the signal 2 candidate causal variant affects breast cancer risk is not mediated through mammographic density or, alternatively, that dense area, as measured here, is unable to capture the association with breast composition that is most relevant to risk. This phenomenon, whereby the association with risk appears to be partially independent of mammographic density, has also been observed for the 10q21.2 breast cancer locus ${ }^{4}$.

SNPs in the ESR1 region have previously been reported to be associated with bone mineral density ${ }^{28,29}$. These include SNPs within signal 1 (rs6930633, $r^{2}=0.73$ with rs3757322) and signal 3 (rs2982575, $r^{2}=0.57$ with $r 851984$ ), although the SNP with the most significant reported association with bone density measures, rs4870044, was not associated with breast cancer risk $\left(P>1 \times 10^{-4}\right)$ in our analysis nor correlated with any signal-representative SNPs $\left(r^{2}<0.06\right)$. Similarly, SNP rs6933669, recently reported as associated with age at menarche ${ }^{30}$, is uncorrelated with these five signals $\left(r^{2}<0.02\right)$ and was not associated with breast cancer $(P=0.1)$. Thus, although there is a known relationship between age at menarche and breast cancer risk, these phenotypes do not appear to share candidate causal variants in this region.

Our findings help address the question of the role of ER $\alpha$ in establishing breast cancer. Notably, the candidate causal SNPs identified here all increase risks of both $\mathrm{ER}^{+}$and $\mathrm{ER}^{-}$tumor subtypes by varying degrees. $\mathrm{ER} \alpha$ is a ligand-activated transcription factor that mediates the effect of estrogen through altering gene expression, and the links between estrogen, ER $\alpha$ and $\mathrm{ER}^{+}$breast cancer are well documented, with adjuvant endocrine therapy considered standard treatment for $\mathrm{ER}^{+}$, early-stage breast cancer. Other studies have also reported $6 \mathrm{q} 25$ associations with $\mathrm{ER}^{-}$subtypes $^{1,2,5}$, but the mechanisms by which $\mathrm{ER}^{-}$tumors develop are still debated. There is speculation that $\mathrm{ER}^{-}$tumors may arise from $\mathrm{ER}^{+}$precursors by potentially reversible mechanisms, and our findings may lend support to this hypothesis. However, several recent studies have indicated that most tumors in $B R C A 1$ mutation carriers arise from $\mathrm{ER}^{-}$luminal progenitor cells; thus, estrogen may be working indirectly through paracrine regulation in the mammary epithelium, possibly stimulating the Notch or epidermal growth factor receptor (EGFR) signaling pathways of adjacent $\mathrm{ER}^{+}$cells ${ }^{31,32}$. Our analyses unexpectedly suggested that, whereas signals 1-4 increased risks of all $\mathrm{ER}^{-}$tumor subtypes, the signal 5 candidate causal variant increased risks of $\mathrm{ER}^{-} \mathrm{HER} 2^{+}$breast cancer subtypes but not of triple-negative tumor development or of tumors in BRCA1 mutation carriers (Table 1). This further complicates present understanding and underlines the need for further studies to address this issue.

Collectively, our evidence supports a hypothesis that ESR 1 is the major target gene of the enhancer and silencer elements in which we have identified candidate causal variants. In addition to ESR1, we provide evidence that the regions overlapping signals 1-4 cooperatively regulate $R M N D 1$, raising the possibility that candidate causal SNPs act by altering both ESR1 and RMND1 expression. RMND1 (required for meiotic nuclear division 1; C6orf96) has not been well characterized but is reported to localize to mitochondria and be involved in mitochondrial translation ${ }^{33}$. We additionally identified enhancer activity and chromatin interactions with two other genes, $A R M T 1$ and CCDC170, but the actions of the candidate causal SNPs on these genes remain unclear. ARMT1 encodes Armt1, a protein carboxyl methyltransferase that targets PCNA and differentially regulates cancer cell survival in response to DNA damage ${ }^{34}$. Nothing is known about the function of CCDC170 (coiled-coil domain-containing protein 170), but recurrent ESR1-CCDC170 rearrangements have been characterized in an aggressive subset of $\mathrm{ER}^{+}$breast cancers ${ }^{35}$. A recent study also showed that higher CCDC170 expression correlated with ER negativity, highly proliferative features and worse clinical outcomes ${ }^{36}$. There are some data to suggest that these genes may cooperatively contribute to the increased proliferative capacity of $\mathrm{ER}^{+}$tumors ${ }^{20}$, and it is tempting to speculate that these may be additional target genes for the candidate causal variants at a subset of the five signals identified here and perhaps responsible for their differential phenotype associations. A greater understanding of these genes may also provide novel targets for breast cancer prevention or therapies.

URLs. 1000 Genomes Project, http://www.1000genomes.org/; Breast Cancer Association Consortium (BCAC), http://ccge.medschl.cam. ac.uk/consortia/bcac/index.html; Consortium of Investigators of Modifiers of BRCA1 and BRCA2 (CIMBA), http://ccge.medschl. cam.ac.uk/consortia/cimba/index.html; Collaborative Oncological Gene-environment Study (COGS), http://www.cogseu.org/; iCOGS, http://ccge.medschl.cam.ac.uk/research/consortia/icogs/; SNAP, https://www.broadinstitute.org/mpg/snap/; The Cancer Genome Atlas (TCGA), https://tcga-data.nci.nih.gov/; Cancer Genomics Hub (CGHub), https://cghub.ucsc.edu/; eMAP, http://www.bios.unc. edu/ weisun/software.htm.

\section{METHODS}

Methods and any associated references are available in the online version of the paper.

Accession codes. The relevant SNP genotype data underpinning these analyses can be accessed by applying to the BCAC and CIMBA consortia (see URLs).

Note: Any Supplementary Information and Source Data files are available in the online version of the paper.

\section{ACKNOWLEDGMENTS}

We thank all the individuals who took part in these studies and all the researchers, clinicians, technicians and administrative staff who have enabled this work to be carried out. This study would not have been possible without the contributions of the following: A. Berchuck (OCAC), R.A. Eeles, A.A. Al Olama, Z. Kote-Jarai and S. Benlloch (PRACTICAL), C. Luccarini and the staff of the Centre for Genetic Epidemiology Laboratory, the staff of the CNIO genotyping unit, D.C. Tessier, F. Bacot, D. Vincent, S. LaBoissière, F. Robidoux and the staff of the McGill University and Génome Québec Innovation Centre, S.F. Nielsen, B.G. Nordestgaard and the staff of the Copenhagen DNA laboratory, and J.M. Cunningham, S.A. Windebank, C.A. Hilker, J. Meyer and the staff of the Mayo Clinic Genotyping Core Facility. Normal human tissues from the Susan G. Komen for the Cure Tissue Bank at the Indiana University Simon Cancer Center (Indianapolis) were used in this study. We thank the contributors, including Indiana University who collected samples used in this study, as well as the donors and their families, whose help and participation made this work possible. We also acknowledge National Institute for Health Research (NIHR) 
support to the Royal Marsden Biomedical Research Centre. Funding for the iCOGS infrastructure came from the European Community's Seventh Framework Programme under grant agreement 223175 (HEALTH-F2-2009-223175) (COGS), Cancer Research UK (C1287/A10118, C1287/A10710, C12292/A11174, C1281/A12014, C5047/A8384, C5047/A15007, C5047/A10692 and C8197/A16565), the US National Institutes of Health (NIH; CA128978, CA192393, CA116167, CA176785 and an NIH Specialized Program of Research Excellence (SPORE) in Breast Cancer (CA116201)) and Post-Cancer GWAS initiative (1U19 CA148537, 1 U19 CA148065 and 1U19 CA148112, the GAME-ON initiative), the US Department of Defense (W81XWH-10-1-0341), the Canadian Institutes of Health Research (CIHR) for the CIHR Team in Familial Risks of Breast Cancer, the Komen Foundation for the Cure, the Breast Cancer Research Foundation and the Ovarian Cancer Research Fund. Full acknowledgments are given in the Supplementary Note.

\section{AUTHOR CONTRIBUTIONS}

Manuscript writing group: A.M.D., K. Michailidou, K.B.K., D. Thompson, J.D.F., K.A.P., J. Beesley, C.S.H., G.C.-T., A.C.A., D.F.E. and S.L.E. Locus SNP selection: A.M.D., C.S.H. and E.D. iCOGS genotyping, genotype calling and quality control: A.M.D., J. Beesley, C.S.H., M.G., G.C.-T., K.A.P. and D.F.E. Imputation: K. Michailidou, K.B.K., A.C.A. and D.F.E. Statistical analyses and programming: K. Michailidou, K.B.K., A.C.A. and D.F.E. Functional analysis and bioinformatics: S.L.E., J.D.F., K.M.H., S. Kaufmann, H.S., M.M.M., J.S.L., E.L.-K., M. Hills, M.J., S.D., J. Beesley, S. Kar, N.A.S.-A., R.C.S., S.C. and S.N. COGS coordination: P.H., D.F.E., J. Beesley and A.M.D. BCAC coordination: D.F.E., G.C.-T., P.D.P.P. and J. Stone. BCAC data management: M.K.B. and Q.W. CIMBA coordination: A.C.A., G.C.-T., J. Stone and F.J.C. CIMBA data management: L.M. and D.B. MODE coordination: D. Thompson, C.V. and F.J.C. Provided participant samples and phenotype information and read and approved the manuscript: A.M.D., K. Michailidou, K.B.K., D. Thompson, J.D.F., J. Beesley, C.S.H., S. Kar, K.A.P., E.L.-K., E.D., D.B., N.A.S.-A., R.C.S., K.M.H., S. Kaufmann, H.S., M.M.M., J.S.L., M. Hills, M.J., S.D., S.C., M.K.B., J.D., Q.W., J.L.H., M.C.S., A. Broeks, M.K.S., A. Lophatananon, K. Muir, M.W.B., P.A.F., I.d.-S.-S., J.P., E.J.S., I.T., B. Burwinkel, F.M., P.G., T.T., S.E.B., H.F., A.G.-N., J.I.A.P., H.A.-C., L.E., V.A., H. Brenner, A. Meindl, R.K.S., H. Brauch, U.H., K.A., C.B., H.I., K. Matsuo, N.B., T.D., A. Lindblom, S. Margolin, V.-M.K., A. Mannermaa, C.T., A.H.W., D.L., H.W., J.C.-C., A.R., P.P., P.R., J.E.O., G.G.G., R.L.M., C.A.H., B.E.H., M.S.G., S.H.T., C.H.Y., S.N., A.-L.B.-D., V.K., J. Long, W.Z., K.P., R.W., I.L.A., J.A.K., P.D., C. Seynaeve, J.F., M.E.S., K.C., H.D., A.Hollestelle, A.M.W.v.d.O., K.H., Y.-T.G., X.-O.S., A.C., S.S.C., W.B., Q.C., B.J.P., M.S., J.-Y.C., D.K., S.C.L., M. Hartman, M. Kabisch, D. Torres, A.J., J. Lubinski, P.B., S.S., C.B.A., A.E.T., C.-Y.S., P.-E.W., N.O., A.S., L.M., S.H., A. Lee, M. Kapuscinski, E.M.J., M.B.T., M.B.D., D.E.G., S.S.B., R.J., L.T., N.T., C.M.D., E.J.v.R., S.L.N., B.E., T.V.O.H., A.O., J. Benitez, R.R., J.N.W., B. Bonanni, B.P., S. Manoukian, L.P., L.O., I.K., P.A., J. Garber, M.U.R., D.F., L.I., S.E., A.K.G., N.A., D.N., K.R., N.B.-M., C. Sagne, D.S.-L., F.D., O.M.S., S. Mazoyer, C.I., K.B.M.C., K.D.L., M.d.l.H., T.C., H.N., S. Khan, A.R.M., M.J.H., M.A.R., A.K., E.O., O.D., J. Brunet, M.A.P., J. Gronwald, T.H., R.B.B., R. Laframboise, P.S., M.M., S.A., M.R.T., S.K.P., N.L., F.J.C., M. Tischkowitz, L.F., J.V., K.O., C.F.S., C.R., C.M.P., M.H.G., P.L.M., G.R., E.N.I., P.J.H., K.-A.P., M.P., A.M.M., G.G., A. Bojesen, M. Thomassen, M.A.C., S.-Y.Y., E.F., Y.L., A. Borg, A.v.W., H.E., J.R., O.I.O., P.A.G., R.L.N., S.A.G., K.L.N., S.M.D., B.K.A., G. Mitchell, B.Y.K., J. Lester, G. Maskarinec, C.W., C. Scott, J. Stone, C.A., R.T., R. Luben, K.-T.K., Å.Helland, V.H., M.D., P.D.P.P., J. Simard, P.H., M.G.-C., C.V., G.C.-T., A.C.A., D.F.E. and, S.L.E.

\section{COMPETING FINANCIAL INTERESTS}

The authors declare no competing financial interests.

Reprints and permissions information is available online at http://www. nature.com/ reprints/index.html.

1. Zheng, W. et al. Genome-wide association study identifies a new breast cancer susceptibility locus at 6q25.1. Nat. Genet. 41, 324-328 (2009).

2. Turnbull, C. et al. Genome-wide association study identifies five new breast cancer susceptibility loci. Nat. Genet. 42, 504-507 (2010)

3. Antoniou, A.C. et al. Common alleles at $6 \mathrm{q} 25.1$ and $1 \mathrm{p} 11.2$ are associated with breast cancer risk for BRCA1 and BRCA2 mutation carriers. Hum. Mol. Genet. 20, 3304-3321 (2011).

4. Lindström, S. et al. Common variants in ZNF365 are associated with both mammographic density and breast cancer risk. Nat. Genet. 43, 185-187 (2011).

5. Stacey, S.N. et al. Ancestry-shift refinement mapping of the C6orf97-ESR1 breast cancer susceptibility locus. PLoS Genet. 6, e1001029 (2010).

6. Hein, R. et al. Comparison of $6 q 25$ breast cancer hits from Asian and European genome wide association studies in the Breast Cancer Association Consortium (BCAC). PLoS One 7, e42380 (2012).
7. Edwards, S.L., Beesley, J., French, J.D. \& Dunning, A.M. Beyond GWASs: illuminating the dark road from association to function. Am. J. Hum. Genet. 93, 779-797 (2013).

8. Mavaddat, N. et al. Pathology of breast and ovarian cancers among BRCA1 and $B R C A 2$ mutation carriers: results from the Consortium of Investigators of Modifiers of BRCA1/2 (CIMBA). Cancer Epidemiol. Biomarkers Prev. 21, 134-147 (2012).

9. Spencer, A.V., Cox, A. \& Walters, K. Comparing the efficacy of SNP filtering methods for identifying a single causal SNP in a known association region. Ann. Hum. Genet. 78, 50-61 (2014).

10. Cai, Q. et al. Replication and functional genomic analyses of the breast cancer susceptibility locus at 6q25.1 generalize its importance in women of Chinese, Japanese, and European ancestry. Cancer Res. 71, 1344-1355 (2011).

11. Li, Q. et al. Integrative eQTL-based analyses reveal the biology of breast cancer risk loci. Cell 152, 633-641 (2013).

12. Corradin, 0 . et al. Combinatorial effects of multiple enhancer variants in linkage disequilibrium dictate levels of gene expression to confer susceptibility to common traits. Genome Res. 24, 1-13 (2014).

13. Hnisz, D. et al. Super-enhancers in the control of cell identity and disease. Cell 155, 934-947 (2013).

14. French, J.D. et al. Functional variants at the $11 \mathrm{q} 13$ risk locus for breast cancer regulate cyclin D1 expression through long-range enhancers. Am. J. Hum. Genet. 92, 489-503 (2013)

15. Ghoussaini, M. et al. Evidence that breast cancer risk at the 2q35 locus is mediated through IGFBP5 regulation. Nat. Commun. 4, 4999 (2014).

16. Glubb, D.M. et al. Fine-scale mapping of the $5 q 11.2$ breast cancer locus reveals at least three independent risk variants regulating MAP3K1. Am. J. Hum. Genet. 96, 5-20 (2015)

17. Cowper-Sal-lari, R. et al. Breast cancer risk-associated SNPs modulate the affinity of chromatin for FOXA1 and alter gene expression. Nat. Genet. 44, 1191-1198 (2012).

18. Ward, L.D. \& Kellis, M. HaploReg: a resource for exploring chromatin states, conservation, and regulatory motif alterations within sets of genetically linked variants. Nucleic Acids Res. 40, D930-D934 (2012).

19. Grabe, N. AliBaba2: context specific identification of transcription factor binding sites. In Silico Biol. 2, S1-S15 (2002).

20. Dunbier, A.K. et al. ESR1 is co-expressed with closely adjacent uncharacterised genes spanning a breast cancer susceptibility locus at 6q25.1. PLoS Genet. 7, e1001382 (2011).

21. Antoniou, A.C. et al A locus on $19 p 13$ modifies risk of breast cancer in BRCA mutation carriers and is associated with hormone receptor-negative breast cancer in the general population. Nat. Genet. 42, 885-892 (2010).

22. Haiman, C.A. et al. A common variant at the TERT-CLPTM1L locus is associated with estrogen receptor-negative breast cancer. Nat. Genet. 43, 1210-1214 (2011).

23. McCormack, V.A. \& dos Santos Silva, I. Breast density and parenchymal patterns as markers of breast cancer risk: a meta-analysis. Cancer Epidemiol. Biomarkers Prev. 15, 1159-1169 (2006).

24. Varghese, J.S. et al. Mammographic breast density and breast cancer: evidence of a shared genetic basis. Cancer Res. 72, 1478-1484 (2012).

25. Crandall, C.J. et al. Sex steroid metabolism polymorphisms and mammographic density in pre- and early perimenopausal women. Breast Cancer Res. 11, R51 (2009).

26. Lindström, S. et al. Genome-wide association study identifies multiple loci associated with both mammographic density and breast cancer risk. Nat. Commun. 5, 5303 (2014).

27. Stone, J. et al. Novel associations between common breast cancer susceptibility variants and risk-predicting mammographic density measures. Cancer Res. 75 2457-2467 (2015).

28. Estrada, K. et al. Genome-wide meta-analysis identifies 56 bone mineral density loci and reveals 14 loci associated with risk of fracture. Nat. Genet. 44, 491-501 (2012).

29. Koller, D.L. et al. Meta-analysis of genome-wide studies identifies WNT16 and ESR 1 SNPs associated with bone mineral density in premenopausal women. J. Bone Miner. Res. 28, 547-558 (2013).

30. Perry, J.R et al. Parent-of-origin-specific allelic associations among 106 genomic loci for age at menarche. Nature 514, 92-97 (2014).

31. Lim, E. et al. Aberrant luminal progenitors as the candidate target population for basal tumor development in BRCA1 mutation carriers. Nat. Med. 15, 907-913 (2009).

32. Molyneux, G. et al. BRCA1 basal-like breast cancers originate from luminal epithelia progenitors and not from basal stem cells. Cell Stem Cell 7, 403-417 (2010).

33. Janer, A. et al. An RMND1 mutation causes encephalopathy associated with multiple oxidative phosphorylation complex deficiencies and a mitochondrial translation defect. Am. J. Hum. Genet. 91, 737-743 (2012).

34. Perry, J.J. et al. Human C6orf21 1 encodes Armt1, a protein carboxyl methyltransferase that targets PCNA and is linked to the DNA damage response. Cell Rep. 10, 1288-1296 (2015)

35. Veeraraghavan, J. et al. Recurrent ESR1-CCDC170 rearrangements in an aggressive subset of oestrogen receptor-positive breast cancers. Nat. Commun. 5, 4577 (2014).

36. Yamamoto-Ibusuki, M. et al. C6ORF97-ESR1 breast cancer susceptibility locus: influence on progression and survival in breast cancer patients. Eur. J. Hum. Genet 23, 949-956 (2015). 
Alison M Dunning1,198, Kyriaki Michailidou ${ }^{2,198}$, Karoline B Kuchenbaecker ${ }^{2,198}$, Deborah Thompson ${ }^{2,198}$, Juliet D French ${ }^{3,198}$, Jonathan Beesley ${ }^{3,198}$, Catherine S Healey ${ }^{1,198}$, Siddhartha Kar ${ }^{1}$, Karen A Pooley ${ }^{2}$, Elena Lopez-Knowles ${ }^{4,5}$, Ed Dicks ${ }^{1}$, Daniel Barrowdale ${ }^{2}$, Nicholas A Sinnott-Armstrong ${ }^{6}$, Richard C Sallari ${ }^{7}$, Kristine M Hillman ${ }^{3}$, Susanne Kaufmann ${ }^{3}$, Haran Sivakumaran ${ }^{3}$, Mahdi Moradi Marjaneh $^{3}$, Jason S Lee ${ }^{3}$, Margaret Hills $^{5}$, Monika Jarosz ${ }^{4,5}$, Suzie Drury ${ }^{4,5}$, Sander Canisius ${ }^{8}$, Manjeet K Bolla ${ }^{2}$, Joe Dennis ${ }^{2}$, Qin Wang ${ }^{2}$, John L Hopper ${ }^{9}$, Melissa C Southey ${ }^{10}$, Annegien Broeks ${ }^{8}$, Marjanka K Schmidt ${ }^{8}$, Artitaya Lophatananon ${ }^{11}$, Kenneth Muir ${ }^{11,12}$, Matthias W Beckmann ${ }^{13}$, Peter A Fasching ${ }^{13,14}$, Isabel dos-Santos-Silva ${ }^{15}$, Julian Peto ${ }^{15}$, Elinor J Sawyer ${ }^{16}$, Ian Tomlinson ${ }^{17,18}$, Barbara Burwinkel ${ }^{19,20}$, Frederik Marme ${ }^{21,22}$, Pascal Guénel ${ }^{23,24}$, Thérèse Truong ${ }^{23,24}$, Stig E Bojesen ${ }^{25-27}$, Henrik Flyger ${ }^{28}$, Anna González-Neira ${ }^{29}$, Jose I A Perez ${ }^{30}$, Hoda Anton-Culver ${ }^{31}$, Lee Eunjung ${ }^{32}$, Volker Arndt ${ }^{33}$, Hermann Brenner ${ }^{33,34}$, Alfons Meindl ${ }^{35}$, Rita K Schmutzler ${ }^{36-38}$, Hiltrud Brauch ${ }^{34,39,40}$, Ute Hamann ${ }^{41}$, Kristiina Aittomäki ${ }^{42}$, Carl Blomqvist ${ }^{43}$, Hidemi Ito $^{44}$, Keitaro Matsuo ${ }^{45}$, Natasha Bogdanova ${ }^{46}$, Thilo Dörk ${ }^{47}$, Annika Lindblom ${ }^{48}$, Sara Margolin ${ }^{49}$, Veli-Matti Kosma ${ }^{50-52}$, Arto Mannermaa ${ }^{50-52}$, Chiu-chen Tseng ${ }^{32}$, Anna H Wu ${ }^{32}$, Diether Lambrechts ${ }^{53,54}$, Hans Wildiers ${ }^{55}$, Jenny Chang-Claude ${ }^{56,57}$, Anja Rudolph ${ }^{56}$, Paolo Peterlongo ${ }^{58}$, Paolo Radice ${ }^{59}$, Janet E Olson ${ }^{60}$, Graham G Giles 9,61 , Roger L Milne ${ }^{9,61}$, Christopher A Haiman ${ }^{32}$, Brian E Henderson ${ }^{32}$, Mark S Goldberg ${ }^{62,63}$, Soo $\mathrm{H} \mathrm{Teo}^{64,65}$, Cheng Har Yip ${ }^{65}$, Silje Nord ${ }^{66}$, Anne-Lise Borresen-Dale ${ }^{66,67}$, Vessela Kristensen ${ }^{66-68}$, Jirong Long ${ }^{69}$, Wei Zheng ${ }^{69}$, Katri Pylkäs ${ }^{70,71}$, Robert Winqvist ${ }^{70,71}$, Irene L Andrulis ${ }^{72,73}$, Julia A Knight ${ }^{74,75}$, Peter Devilee $^{76,77}$, Caroline Seynaeve ${ }^{78}$, Jonine Figueroa ${ }^{79}$, Mark E Sherman ${ }^{79}$, Kamila Czene ${ }^{80}$, Hatef Darabi ${ }^{80}$, Antoinette Hollestelle ${ }^{78}$, Ans M W van den Ouweland ${ }^{81}$, Keith Humphreys ${ }^{80}$, Yu-Tang Gao ${ }^{82}$, Xiao-Ou Shu ${ }^{69}$, Angela Cox ${ }^{83}$, Simon S Cross ${ }^{84}$, William Blot ${ }^{69,85}$, Qiuyin Cai ${ }^{69}$, Maya Ghoussaini ${ }^{1}$, Barbara J Perkins ${ }^{1}$, Mitul Shah ${ }^{1}$, Ji-Yeob Choi ${ }^{86,87}$, Daehee Kang ${ }^{86-88}$, Soo Chin Lee ${ }^{89,90}$, Mikael Hartman ${ }^{91,92}$, Maria Kabisch ${ }^{41}$, Diana Torres ${ }^{41,93}$, Anna Jakubowska ${ }^{94}$, Jan Lubinski ${ }^{94}$, Paul Brennan ${ }^{95}$, Suleeporn Sangrajrang ${ }^{96}$, Christine B Ambrosone ${ }^{97}$, Amanda E Toland ${ }^{98}$, Chen-Yang Shen ${ }^{99,100}$, Pei-Ei Wu ${ }^{100}$, Nick Orr ${ }^{101}$, Anthony Swerdlow ${ }^{102,103}$, Lesley McGuffog ${ }^{2}$, Sue Healey ${ }^{3}$, Andrew Lee ${ }^{2}$, Miroslav Kapuscinski ${ }^{104}$, Esther M John ${ }^{105}$, Mary Beth Terry ${ }^{106}$, Mary B Daly ${ }^{107}$, David E Goldgar ${ }^{108}$, Saundra S Buys ${ }^{109}$, Ramunas Janavicius ${ }^{110}$, Laima Tihomirova ${ }^{111}$, Nadine Tung ${ }^{112}$, Cecilia M Dorfling ${ }^{113}$, Elizabeth J van Rensburg ${ }^{113}$, Susan L Neuhausen ${ }^{114}$, Bent Ejlertsen ${ }^{115}$, Thomas V O Hansen ${ }^{116}$, Ana Osorio ${ }^{117,118}$, Javier Benitez ${ }^{117-119}$, Rachel Rando ${ }^{120}$, Jeffrey N Weitzel ${ }^{121}$, Bernardo Bonanni ${ }^{122}$, Bernard Peissel ${ }^{123}$, Siranoush Manoukian ${ }^{123}$, Laura Papi ${ }^{124}$, Laura Ottini ${ }^{125}$, Irene Konstantopoulou ${ }^{126}$, Paraskevi Apostolou ${ }^{126}$, Judy Garber ${ }^{127}$, Muhammad Usman Rashid ${ }^{41,128}$, Debra Frost ${ }^{2}$, EMBRACE ${ }^{129}$, Louise Izatt ${ }^{130}$, Steve Ellis ${ }^{2}$, Andrew K Godwin ${ }^{131}$, Norbert Arnold ${ }^{132}$, Dieter Niederacher ${ }^{133}$, Kerstin Rhiem ${ }^{134}$, Nadja Bogdanova-Markov ${ }^{135}$, Charlotte Sagne ${ }^{136}$, Dominique Stoppa-Lyonnet $^{137,138}$, Francesca Damiola ${ }^{136}$, GEMO Study Collaborators ${ }^{129}$, Olga M Sinilnikova ${ }^{136,139}$, Sylvie Mazoyer ${ }^{136}$, Claudine Isaacs ${ }^{140}$, Kathleen B M Claes ${ }^{141}$, Kim De Leeneer ${ }^{141}$, Miguel de la Hoya ${ }^{142}$, Trinidad Caldes ${ }^{142}$, Heli Nevanlinna ${ }^{143}$, Sofia Khan ${ }^{143}$, Arjen R Mensenkamp ${ }^{144}$, HEBON ${ }^{129}$, Maartje J Hooning ${ }^{145}$, Matti A Rookus ${ }^{146}$, Ava Kwong ${ }^{147,148}$, Edith Olah ${ }^{149}$, Orland Diez ${ }^{150}$, Joan Brunet ${ }^{151}$, Miquel Angel Pujana ${ }^{152}$, Jacek Gronwald ${ }^{94}$, Tomasz Huzarski ${ }^{94}$, Rosa B Barkardottir ${ }^{153}$, Rachel Laframboise ${ }^{154}$, Penny Soucy ${ }^{155}$, Marco Montagna ${ }^{156}$, Simona Agata ${ }^{156}$, Manuel R Teixeira ${ }^{157,158}$, kConFab Investigators ${ }^{129}$, Sue Kyung Park ${ }^{86-88}$, Noralane Lindor ${ }^{60}$, Fergus J Couch ${ }^{60,159}$, Marc Tischkowitz ${ }^{160}$, Lenka Foretova ${ }^{161}$, Joseph Vijai ${ }^{162}$, Kenneth Offit ${ }^{162}$, Christian F Singer ${ }^{163}$, Christine Rappaport ${ }^{163}$, Catherine M Phelan ${ }^{164}$, Mark H Greene ${ }^{165}$, Phuong L Mai ${ }^{165}$, Gad Rennert ${ }^{166,167}$, Evgeny N Imyanitov ${ }^{168}$, Peter J Hulick ${ }^{169}$, Kelly-Anne Phillips ${ }^{170}$, Marion Piedmonte ${ }^{171}$, Anna Marie Mulligan ${ }^{172,173}$, Gord Glendon ${ }^{72}$, Anders Bojesen ${ }^{174}$, Mads Thomassen ${ }^{175}$, Maria A Caligo ${ }^{176}$, Sook-Yee Yoon ${ }^{64,177}$, Eitan Friedman ${ }^{178}$, Yael Laitman ${ }^{178}$, Ake Borg ${ }^{179}$, Anna von Wachenfeldt ${ }^{49}$, Hans Ehrencrona ${ }^{180,181}$, Johanna Rantala ${ }^{182}$, Olufunmilayo I Olopade ${ }^{183}$, Patricia A Ganz ${ }^{184}$, Robert L Nussbaum ${ }^{185}$, Simon A Gayther ${ }^{32}$, Katherine L Nathanson ${ }^{186}$, Susan M Domchek ${ }^{186}$, Banu K Arun ${ }^{187}$, Gillian Mitchell ${ }^{188,189}$, Beth Y Karlan ${ }^{190}$, Jenny Lester ${ }^{190}$, Gertraud Maskarinec ${ }^{191}$, Christy Woolcott ${ }^{192}$, Christopher Scott ${ }^{60}$, Jennifer Stone ${ }^{193}$, Carmel Apicella9, Rulla Tamimi ${ }^{194-196}$, Robert Luben ${ }^{197}$, Kay-Tee Khaw ${ }^{197}$, Åslaug Helland ${ }^{66}$, Vilde Haakensen ${ }^{66}$, Mitch Dowsett ${ }^{4,5}$, Paul D P Pharoah ${ }^{1,2}$, Jacques Simard ${ }^{155}$, Per Hall ${ }^{80}$, Montserrat García-Closas ${ }^{101,102}$, Celine Vachon ${ }^{60}$, Georgia Chenevix-Trench ${ }^{3}$, Antonis C Antoniou ${ }^{2,199}$, Douglas F Easton ${ }^{1,2,199}$ \& Stacey L Edwards 3,199 
${ }^{1}$ Centre for Cancer Genetic Epidemiology, Department of Oncology, University of Cambridge, Cambridge, UK. ${ }^{2}$ Centre for Cancer Genetic Epidemiology, Department of Public Health and Primary Care, University of Cambridge, Cambridge, UK. ${ }^{3}$ Cancer Division, QIMR Berghofer Medical Research Institute, Brisbane, Queensland, Australia. ${ }^{4}$ Breast Cancer Research, Breakthrough Breast Cancer Research Centre, London, UK. ${ }^{5}$ Academic Biochemistry, Royal Marsden Hospital, London, UK. ${ }^{6}$ Department of Genetics, Stanford University School of Medicine, Stanford, California, USA. ${ }^{7}$ Computer Science and Artificial Intelligence Laboratory, Massachusetts Institute of Technology, Cambridge, Massachusetts, USA. ${ }^{8}$ Netherlands Cancer Institute, Antoni van Leeuwenhoek Hospital, Amsterdam, the Netherlands. ${ }^{9}$ Centre for Epidemiology and Biostatistics, School of Population and Global Health, University of Melbourne, Melbourne, Victoria, Australia. ${ }^{10}$ Department of Pathology, University of Melbourne, Melbourne, Victoria, Australia. ${ }^{11}$ Division of Health Sciences, Warwick Medical School, Warwick University, Coventry, UK. ${ }^{12}$ Institute of Population Health, University of Manchester, Manchester, UK. ${ }^{13}$ Department of Gynecology and Obstetrics, University Hospital Erlangen, Friedrich Alexander University Erlangen-Nuremberg, Comprehensive Cancer Center Erlangen-Nuremberg Metropolitan Region, Erlangen, Germany. ${ }^{14}$ Department of Medicine, Division of Hematology and Oncology, David Geffen School of Medicine, University of California, Los Angeles, Los Angeles, California, USA. ${ }^{15}$ Department of NonCommunicable Disease Epidemiology, London School of Hygiene and Tropical Medicine, London, UK. ${ }^{16}$ Research Oncology, Division of Cancer Studies, King's College London, Guy's Hospital, London, UK. ${ }^{17}$ Wellcome Trust Centre for Human Genetics, University of Oxford, Oxford, UK. ${ }^{18}$ Oxford Biomedical Research Centre, University of Oxford, Oxford, UK. ${ }^{19}$ Division of Molecular Genetic Epidemiology, German Cancer Research Center, Heidelberg, Germany. ${ }^{20}$ Molecular Epidemiology Group, German Cancer Research Center, Heidelberg, Germany. ${ }^{21}$ National Center for Tumor Diseases, University of Heidelberg, Heidelberg, Germany. ${ }^{22}$ Department of Obstetrics and Gynecology, University of Heidelberg, Heidelberg, Germany. ${ }^{23}$ Environmental Epidemiology of Cancer, Center for Research in Epidemiology and Population Health, INSERM, Villejuif, France. ${ }^{24}$ University Paris-Sud, Villejuif, France. ${ }^{25}$ Copenhagen General Population Study, Herlev Hospital, Copenhagen University Hospital, Herlev, Denmark. ${ }^{26}$ Department of Clinical Biochemistry, Herlev Hospital, Copenhagen University Hospital, Herlev, Denmark. ${ }^{27}$ Faculty of Health and Medical Sciences, University of Copenhagen, Copenhagen, Denmark. ${ }^{28}$ Department of Breast Surgery, Herlev Hospital, Copenhagen University Hospital, Herlev, Denmark. ${ }^{29}$ Human Cancer Genetics Program, Spanish National Cancer Centre (CNIO), Madrid, Spain. ${ }^{30}$ Servicio de Cirugía General y Especialidades, Hospital Monte Naranco, Oviedo, Spain. ${ }^{31}$ Department of Epidemiology, University of California-Irvine, Irvine, California, USA. ${ }^{32}$ Department of Preventive Medicine, Keck School of Medicine, University of Southern California, Los Angeles, California, USA. ${ }^{33}$ Division of Clinical Epidemiology and Aging Research, German Cancer Research Center, Heidelberg, Germany. ${ }^{34}$ German Cancer Consortium, German Cancer Research Center, Heidelberg, Germany. ${ }^{35}$ Department of Gynaecology and Obstetrics, Technical University of Munich, Munich, Germany. ${ }^{36}$ Division of Molecular Gyneco-Oncology, Department of Gynaecology and Obstetrics, University Hospital of Cologne, Cologne, Germany. ${ }^{37}$ Centre of Familial Breast and Ovarian Cancer, University Hospital of Cologne, Cologne, Germany. ${ }^{38}$ Center for Integrated Oncology, University Hospital, Cologne, Germany. ${ }^{39}$ Dr. Margarete Fischer-Bosch Institute of Clinical Pharmacology, Stuttgart, Germany. ${ }^{40}$ University of Tübingen, Tübingen, Germany. ${ }^{41}$ Molecular Genetics of Breast Cancer, German Cancer Research Center, Heidelberg, Germany. ${ }^{42}$ Department of Clinical Genetics, Helsinki University Central Hospital, Helsinki, Finland. ${ }^{43}$ Department of Oncology, Helsinki University Central Hospital, University of Helsinki, Helsinki, Finland. ${ }^{44}$ Division of Epidemiology and Prevention, Aichi Cancer Center Research Institute, Aichi, Japan. ${ }^{45}$ Division of Molecular Medicine, Aichi Cancer Center Research Institute, Nagoya, Japan. ${ }^{46}$ Radiation Oncology Research Unit, Hannover Medical School, Hannover, Germany. ${ }^{47}$ Gynaecology Research Unit, Hannover Medical School, Hannover, Germany. ${ }^{48}$ Department of Molecular Medicine and Surgery, Karolinska Institutet, Stockholm, Sweden. ${ }^{49}$ Department of Oncology-Pathology, Karolinska University Hospital, Stockholm, Sweden. ${ }^{50}$ Cancer Center, Kuopio University Hospital, Kuopio, Finland. ${ }^{51}$ Institute of Clinical Medicine, Pathology and Forensic Medicine, University of Eastern Finland, Kuopio, Finland. ${ }^{52}$ Imaging Center, Department of Clinical Pathology, Kuopio University Hospital, Kuopio, Finland. ${ }^{53}$ Vesalius Research Center, Leuven, Belgium. ${ }^{54}$ Laboratory for Translational Genetics, Department of Oncology, University of Leuven, Leuven, Belgium. ${ }^{55}$ Multidisciplinary Breast Center, Department of General Medical Oncology, University Hospitals Leuven, Leuven, Belgium. 56Division of Cancer Epidemiology, German Cancer Research Center, Heidelberg, Germany. ${ }^{57}$ University Cancer Center Hamburg (UCCH), University Medical Center Hamburg-Eppendorf, Hamburg, Germany. ${ }^{58}$ IFOM, Fondazione Istituto FIRC di Oncologia Molecolare, Milan, Italy. ${ }^{59}$ Unit of Molecular Basis of Genetic Risk and Genetic Testing, Department of Preventive and Predictive Medicine, Fondazione IRCCS Istituto Nazionale dei Tumori, Milan, Italy. ${ }^{60}$ Department of Health Sciences Research, Mayo Clinic, Rochester, Minnesota, USA. ${ }^{61}$ Cancer Epidemiology Centre, Cancer Council Victoria, Melbourne, Victoria, Australia. ${ }^{62}$ Department of Medicine, McGill University, Montreal, Quebec, Canada. ${ }^{63}$ Division of Clinical Epidemiology, Royal Victoria Hospital, McGill University, Montreal, Quebec, Canada. ${ }^{64}$ Cancer Research Initiatives Foundation, Sime Darby Medical Centre, Subang Jaya, Malaysia. ${ }^{65}$ Breast Cancer Research Unit, University Malaya Cancer Research Institute, University Malaya Medical Centre, Kuala Lumpur, Malaysia. ${ }^{66}$ Department of Genetics, Institute for Cancer Research, Oslo University Hospital, Radiumhospitalet, Oslo, Norway. ${ }^{67}$ Institute of Clinical Medicine, University of Oslo, Oslo, Norway. ${ }^{68}$ Department of Clinical Molecular Biology, Oslo University Hospital, University of Oslo, Oslo, Norway. ${ }^{69}$ Division of Epidemiology, Department of Medicine, Vanderbilt-Ingram Cancer Center, Vanderbilt University School of Medicine, Nashville, Tennessee, USA. ${ }^{70}$ Laboratory of Cancer Genetics and Tumor Biology, Department of Clinical Chemistry and Biocenter Oulu, University of Oulu, NordLab Oulu University Hospital, Oulu, Finland. ${ }^{71}$ Laboratory of Cancer Genetics and Tumor Biology, Northern Finland Laboratory Centre NordLab, Oulu, Finland. ${ }^{72}$ Lunenfeld-Tanenbaum Research Institute of Mount Sinai Hospital, Toronto, Ontario, Canada. ${ }^{73}$ Department of Molecular Genetics, University of Toronto, Toronto, Ontario, Canada. ${ }^{74}$ Prosserman Centre for Health Research, Lunenfeld-Tanenbaum Research Institute of Mount Sinai Hospital, Toronto, Ontario, Canada. ${ }^{75}$ Division of Epidemiology, Dalla Lana School of Public Health, University of Toronto, Toronto, Ontario, Canada. ${ }^{76}$ Department of Pathology, Leiden University Medical Center, Leiden, the Netherlands. ${ }^{77}$ Department of Human Genetics, Leiden University Medical Center, Leiden, the Netherlands. ${ }^{78}$ Department of Medical Oncology, Erasmus University Medical Center, Rotterdam, the Netherlands. ${ }^{79}$ Division of Cancer Epidemiology and Genetics, National Cancer Institute, Rockville, Maryland, USA. ${ }^{80}$ Department of Medical Epidemiology and Biostatistics, Karolinska Institutet, Stockholm, Sweden. ${ }^{81}$ Department of Clinical Genetics, Erasmus University Medical Center, Rotterdam, the Netherlands. ${ }^{22}$ Department of Epidemiology, Shanghai Cancer Institute, Shanghai, China. ${ }^{83}$ Sheffield Cancer Research, Department of Oncology, University of Sheffield, Sheffield, UK. ${ }^{84}$ Academic Unit of Pathology, Department of Neuroscience, University of Sheffield, Sheffield, UK. ${ }^{85}$ International Epidemiology Institute, Rockville, Maryland, USA. ${ }^{86}$ Department of Preventive Medicine, Seoul National University College of Medicine, Seoul, Korea. ${ }^{87}$ Department of Biomedical Sciences, Seoul National University College of Medicine, Seoul, Korea. ${ }^{88}$ Cancer Research Institute, Seoul National University College of Medicine, Seoul, Korea. ${ }^{89}$ Department of Haematology-Oncology, National University Health System, Singapore. ${ }^{90} \mathrm{Cancer} \mathrm{Science} \mathrm{Institute} \mathrm{of} \mathrm{Singapore,} \mathrm{National} \mathrm{University} \mathrm{of} \mathrm{Singapore,} \mathrm{Singapore.}{ }^{91}$ Saw Swee Hock School of Public Health, National University of Singapore, Singapore. ${ }^{92}$ Department of Surgery, National University Health System, Singapore. ${ }^{93}$ Institute of Human Genetics, Pontificia Universidad Javerianar, Bogota, Colombia. ${ }^{4}$ Department of Genetics and Pathology, Pomeranian Medical University, Szczecin, Poland. ${ }^{55}$ International Agency for Research on Cancer, Lyon, France. ${ }^{96}$ National Cancer Institute, Bangkok, Thailand. ${ }^{97}$ Roswell Park Cancer Institute, Buffalo, New York, USA. ${ }^{8}$ Department of Molecular Virology, Immunology and Medical Genetics, Comprehensive Cancer Center, The Ohio State University, Columbus, Ohio, USA. ${ }^{99}$ School of Public Health, China Medical University, Taichung, Taiwan. ${ }^{100}$ Taiwan Biobank, Institute of Biomedical Sciences, Academia Sinica, Taipei, Taiwan. ${ }^{101}$ Division of Cancer Studies, Breakthrough Breast Cancer Research Centre, Institute of Cancer Research, London, UK. 102Division of Genetics and Epidemiology, Institute of Cancer Research, London, UK. ${ }^{103}$ Division of Breast Cancer Research, Institute of Cancer Research, London, UK. ${ }^{104}$ Centre for Epidemiology and Biostatistics, University of Melbourne, Melbourne, Victoria, Australia. ${ }^{105}$ Department of Epidemiology, Cancer Prevention Institute of California, Fremont, California, USA. ${ }^{106}$ Department of Epidemiology, Mailman School of Public Health, Columbia University, New York, New York, USA. ${ }^{107}$ Department of Clinical Genetics, Fox Chase Cancer Center, Philadelphia, Pennsylvania, USA. ${ }^{108}$ Department of Dermatology, Huntsman Cancer Institute, University of Utah School of Medicine, Salt Lake City, Utah, USA. ${ }^{109}$ Department of Medicine, Huntsman Cancer Institute, University of Utah School of Medicine, Salt Lake City, Utah, USA. ${ }^{110}$ State Research Institute Centre for Innovative Medicine, Vilnius, Lithuania. ${ }^{111}$ Latvian Biomedical Research and Study Centre, Riga, Latvia. ${ }^{112}$ Department of Medical Oncology, Beth Israel Deaconess Medical Center, Boston, Massachusetts, USA. ${ }^{113}$ Department of Genetics, University of Pretoria, Pretoria, South Africa. ${ }^{114}$ Department of Population Sciences, Beckman Research Institute of City of Hope, Duarte, California, USA. ${ }^{115}$ Department of Oncology, Rigshospitalet, Copenhagen University Hospital, Copenhagen, Denmark. ${ }^{116}$ Center for Genomic Medicine, Rigshospitalet, Copenhagen University Hospital, Copenhagen, Denmark. ${ }^{117}$ Human Genetics Group, Spanish National Cancer Centre (CNIO), Madrid, Spain. ${ }_{118}$ Biomedical Network on Rare Diseases (CIBERER), Madrid, Spain. ${ }^{119}$ Human Genotyping (CEGEN) Unit, Human Cancer Genetics Program, Spanish National Cancer Research Centre (CNIO), Madrid, Spain. ${ }^{120}$ City of Hope Clinical Cancer Genomics Community Research Network, Duarte, California, USA. ${ }^{121}$ Clinical Cancer Genetics, City of Hope, Duarte, California, USA. ${ }^{122}$ Division of Cancer Prevention and Genetics, Istituto Europeo di Oncologia, Milan, Italy. ${ }^{123}$ Unit of Medical Genetics, Department of Preventive and Predictive Medicine, Fondazione Istituto di Ricovero e Cura a Carattere Scientifico, Istituto Nazionale Tumori, Milan, Italy. ${ }^{124}$ Unit of Medical Genetics, Department of Biomedical, Experimental and Clinical Sciences, University of Florence, Florence, Italy. ${ }^{125}$ Department of Molecular 
Medicine, University La Sapienza, Rome, Italy. ${ }^{126}$ Molecular Diagnostics Laboratory, INRASTES (Institute of Nuclear and Radiological Sciences and Technology), National Centre for Scientific Research 'Demokritos', Aghia Paraskevi Attikis, Athens, Greece. ${ }^{127}$ Cancer Risk and Prevention Clinic, Dana-Farber Cancer Institute, Boston, Massachusetts, USA. ${ }^{128}$ Department of Basic Sciences, Shaukat Khanum Memorial Cancer Hospital and Research Centre, Lahore, Pakistan. ${ }^{129} \mathrm{~A}$ full list of members appears in the Supplementary Note. ${ }^{130} \mathrm{Clinical}$ Genetics, Guy's and St. Thomas' National Health Service (NHS) Foundation Trust, London, UK.

${ }_{131}$ Department of Pathology and Laboratory Medicine, University of Kansas Medical Center, Kansas City, Kansas, USA. 132Department of Gynaecology and Obstetrics, University Hospital of Schleswig-Holstein, Campus Kiel, Christian Albrechts University Kiel, Kiel, Germany. ${ }^{133}$ University Düsseldorf, Dusseldorf, Germany.

${ }^{134}$ Centre of Familial Breast and Ovarian Cancer, Department of Gynaecology and Obstetrics and Centre for Integrated Oncology, Center for Molecular Medicine Cologne, University Hospital of Cologne, Cologne, Germany. ${ }^{135}$ Institute of Human Genetics, Münster, Germany. ${ }^{136}$ INSERM U1052, CNRS UMR 5286, Université Lyon, Centre de Recherche en Cancérologie de Lyon, Lyon, France. ${ }^{137}$ Institut Curie, Department of Tumour Biology, Paris, France. 138Université Paris Descartes, Sorbonne Paris Cité, Paris, France. 139Unité Mixte de Génétique Constitutionnelle des Cancers Fréquents, Hospices Civils de Lyon-Centre Léon Bérard, Lyon, France. ${ }^{140}$ Lombardi Comprehensive Cancer Center, Georgetown University, Washington, DC, USA. ${ }^{141}$ Center for Medical Genetics, Ghent University, Ghent, Belgium. ${ }_{142}$ Molecular Oncology Laboratory, Hospital Clinico San Carlos, IdISSC (El Instituto de Investigación Sanitaria del Hospital Clínico San Carlos), Madrid, Spain. ${ }^{143}$ Department of Obstetrics and Gynecology, University of Helsinki and Helsinki University Central Hospital, Helsinki, Finland. ${ }^{144}$ Department of Human Genetics, Radboud University Nijmegen Medical Centre, Nijmegen, the Netherlands. ${ }^{145}$ Department of Medical Oncology, Family Cancer Clinic, Erasmus University Medical Center, Rotterdam, the Netherlands. ${ }^{146}$ Department of Epidemiology, Netherlands Cancer Institute, Amsterdam, the Netherlands. ${ }^{147}$ Hong Kong Hereditary Breast Cancer Family Registry, Cancer Genetics Center, Hong Kong Sanatorium and Hospital, Hong Kong. 148Department of Surgery, University of Hong Kong, Hong Kong. ${ }^{149}$ Department of Molecular Genetics, National Institute of Oncology, Budapest, Hungary. ${ }^{150}$ Oncogenetics Laboratory, Vall d'Hebron Institute of Oncology (VHIO), Vall d'Hebron University Hospital, Barcelona, Spain. ${ }^{151}$ Genetic Counseling Unit, Hereditary Cancer Program, IDIBGI (Institut d'Investigació Biomèdica de Girona), Catalan Institute of Oncology, Girona, Spain. ${ }^{152}$ Breast Cancer and Systems Biology Unit, IDIBELL (Bellvitge Biomedical Research Institute), Catalan Institute of Oncology, Barcelona, Spain. ${ }^{153}$ Department of Pathology, Landspitali University Hospital and Biomedical Centre (BMC), Faculty of Medicine, University of Iceland, Reykjavik, Iceland. ${ }^{154}$ Medical Genetic Division, Centre Hospitalier Universitaire de Québec and Laval University, Quebec City, Quebec, Canada. ${ }^{155}$ Centre Hospitalier Universitaire de Québec and Laval University, Quebec City, Quebec, Canada. ${ }^{156} / \mathrm{mm} u n o l o g y$ and Molecular Oncology Unit, Istituto Oncologico Veneto (IOV), IRCCS (Istituto di Ricovero e Cura a Carattere Scientifico), Padua, Italy. ${ }^{157}$ Department of Genetics, Portuguese Oncology Institute, Porto, Portugal. 158Biomedical Sciences Institute (ICBAS), Porto University, Porto, Portugal. ${ }^{159}$ Department of Laboratory Medicine and Pathology, Mayo Clinic, Rochester, Minnesota, USA. ${ }^{160}$ Program in Cancer Genetics, McGill University, Montreal, Quebec, Canada. ${ }^{161}$ Masaryk Memorial Cancer Institute and Medical Faculty, Masaryk University, Brno, Czech Republic. ${ }^{162}$ Department of Medicine, Memorial Sloan Kettering Cancer Center, New York, New York, USA. ${ }^{163}$ Department of Obstetrics and Gynecology, Comprehensive Cancer Center, Medical University of Vienna, Vienna, Austria. ${ }^{164}$ Department of Cancer Epidemiology, Moffitt Cancer Center, Tampa, Florida, USA. ${ }^{165}$ Clinical Genetics Branch, Division of Cancer Epidemiology and Genetics, National Cancer Institute, US National Institutes of Health, Rockville, Maryland, USA. ${ }^{166}$ Department of Community Medicine and Epidemiology, Carmel Medical Center and B. Rappaport Faculty of Medicine, Haifa, Israel. ${ }^{167} \mathrm{Clalit}$ National Israeli Cancer Control Center, Haifa, Israel. ${ }^{168}$ N.N. Petrov Institute of Oncology, St. Petersburg, Russia. ${ }^{169} \mathrm{Center}$ for Medical Genetics, NorthShore University Health System, Evanston, Illinois, USA. ${ }^{170}$ Division of Cancer Medicine, Peter MacCallum Cancer Centre, East Melbourne, Victoria, Australia. ${ }^{171}$ NRG Oncology, Statistics and Data Management Center, Roswell Park Cancer Institute, Buffalo, New York, USA. ${ }^{172}$ Laboratory Medicine Program, University Health Network, Toronto, Ontario, Canada. ${ }^{173}$ Department of Laboratory Medicine and Pathobiology, University of Toronto, Toronto, Ontario, Canada. ${ }^{174}$ Department of Clinical Genetics, Vejle Hospital, Vejle, Denmark.

${ }^{175}$ Department of Clinical Genetics, Odense University Hospital, Odense, Denmark. ${ }^{176}$ Section of Genetic Oncology, Department of Laboratory Medicine, University of Pisa and University Hospital of Pisa, Pisa, Italy. ${ }^{177}$ University Malaya Cancer Research Institute, Faculty of Medicine, University Malaya Medical Centre, University Malaya, Kuala Lumpur, Malaysia. ${ }^{178}$ Susanne Levy Gertner Oncogenetics Unit, Sheba Medical Center, Tel-Hashomer, Israel. ${ }^{179}$ Department of Oncology, Lund University, Lund, Sweden. ${ }^{180}$ Department of Immunology, Genetics and Pathology, Uppsala University, Uppsala, Sweden. ${ }^{181}$ Department of Clinical Genetics, Lund University Hospital, Lund, Sweden. ${ }^{182}$ Department of Clinical Genetics, Karolinska University Hospital, Stockholm, Sweden. ${ }^{183}$ Center for Clinical Cancer Genetics and Global Health, University of Chicago Medical Center, Chicago, Illinois, USA. ${ }^{184}$ Division of Cancer Prevention and Control Research, Jonsson Comprehensive Cancer Center, University of California Los Angeles School of Medicine and School of Public Health, Los Angeles, California, USA. ${ }^{185}$ Department of Medicine and Genetics, University of California, San Francisco, San Francisco, California, USA. ${ }^{186}$ Abramson Cancer Center, Perelman School of Medicine, University of Pennsylvania, Philadelphia, Pennsylvania, USA. ${ }^{187}$ University of Texas MD Anderson Cancer Center, Houston, Texas, USA. ${ }^{188}$ Familial Cancer Centre, Peter MacCallum Cancer Centre, Melbourne, Victoria, Australia. ${ }^{189}$ Sir Peter MacCallum Department of Oncology, University of Melbourne, Melbourne, Victoria, Australia. 190Women's Cancer Program at the Samuel Oschin Comprehensive Cancer Institute, Cedars-Sinai Medical Center, Los Angeles, California, USA. 191 University of Hawaii Cancer Center, Honolulu, Hawaii, USA. ${ }^{192}$ Department of Obstetrics, Gynaecology and Pediatrics, Dalhousie University, Halifax, Nova Scotia, Canada. ${ }^{193}$ Centre for Genetic Origins of Health and Disease, University of Western Australia, Perth, Western Australia, Australia. ${ }^{194}$ Channing Division of Network Medicine, Department of Medicine, Brigham and Women's Hospital and Harvard Medical School, Boston, Massachusetts, USA. 195Department of Epidemiology, Harvard School of Public Health, Boston, Massachusetts, USA. 196Program in Genetic Epidemiology and Statistical Genetics, Harvard School of Public Health, Boston, Massachusetts, USA. ${ }^{197}$ Clinical Gerontology, Department of Public Health and Primary Care, University of Cambridge, Cambridge, UK. ${ }^{198}$ These authors contributed equally to this work. ${ }^{199}$ These authors jointly supervised this work. Correspondence should be addressed to A.M.D. (amd24@medschl.cam.ac.uk), D.F.E. (dfe20@medschl.cam.ac.uk) or S.L.E. (stacey.edwards@qimrberghofer.edu.au). 


\section{ONLINE METHODS}

Study populations and genotyping. Epidemiological data were obtained from three separate consortia that had all conducted genotyping using the iCOGS array, a custom array comprising approximately 200,000 SNPs. (i) Data on overall breast cancer risk, tumor subtypes and grade came from 50 breast cancer case-control studies participating in BCAC; these comprised 41 studies from populations of European ancestry and 9 studies from populations of East Asian ancestry ${ }^{3}$. Details of the participating studies, genotype calling and quality control are given elsewhere ${ }^{3}$. After quality control exclusions, we analyzed data from 46,451 cases and 42,599 controls of European ancestry and 6,269 cases and 6,624 controls of Asian ancestry. A further 23 SNPs were directly genotyped in two case-control studies (CCHS and SEARCH). The ER status of the primary tumor was available for 34,539 European and 4,972 Asian cases; of these, the tumor was $\mathrm{ER}^{-}$for 7,465 (22\%) European and 1,610 (32\%) Asian cases $^{3}$. (ii) Data on BRCA1 mutation carriers were obtained through CIMBA. Eligibility is restricted to females 18 years or older with pathogenic mutations in BRCA1 or BRCA2. The majority of the participants were sampled through cancer genetics clinics ${ }^{37}$, including some related participants. Fifty-one studies from 25 countries contributed data on BRCA1 mutation carriers who were genotyped using the iCOGS array ${ }^{38}$. After quality control of the phenotypes and genotypes, data were available on 15,252 BRCA1 mutation carriers, of whom 7,797 had been diagnosed with breast cancer, all of European ancestry. Analyses in BRCA1 mutation carriers assessed associations with breast cancer risk. (iii) Mammographic density information was available for 7,025 women from ten studies in BCAC and, in addition, 1,621 women from the Mayo Mammographic Health Study (MMHS). All were additionally participants in the MODE Consortium. Forty-six women were excluded because of missing BMI information, leaving 8,600 women with mammographic density information, relevant covariates and iCOGS genotyping (2,955 breast cancer cases and 5,645 controls). Study details are given in Supplementary Table 14 and in Lindstrom et al. ${ }^{26}$. Mammographic density measurements were performed on digitized analog mammographic films using 'Cumulus' software ${ }^{39}$. This applies a thresholding technique to measure the total area of the breast and the absolute dense area, from which the absolute non-dense area and percent dense area are derived. Dense areas and non-dense areas were converted to $\mathrm{cm}^{2}$ according to the pixel size used in the digitization. Readers blinded to genotype, case status and risk factor data conducted all measures. For cases, mammograms before the diagnosis of breast cancer were used or, where this was not possible, measures from the contralateral breast were used.

SNP selection, genotyping and imputation. We first defined a mapping interval of $\sim 1 \mathrm{Mb}$ (chr. 6: 151,600,000-152,650,000; NCBI Build 37 assembly). We catalogued 2,821 variants with a MAF $>2 \%$ using the 1000 Genomes Project (March 2010 Pilot version 60 CEU project data); of these variants, we selected 277 SNPs correlated with the 3 previously reported associated SNPs (rs2046210 (ref. 1), rs3757318 (ref. 2) and rs3020314 (ref. 40)) at $r^{2}>0.1$, plus a set of 698 SNPs designed to tag all remaining SNPs with $r^{2}>0.9$. Of the SNPs, 902 that passed quality control were included in this analysis. After completion of iCOGS genotyping, this initial set was supplemented with a further 23 SNPs selected from the October 2010 (Build 37) release of the 1000 Genomes Project, to improve coverage. These SNPs were genotyped in two large BCAC studies (CCHS and SEARCH) comprising 12,273 cases and controls, using a Fluidigm array according to the manufacturer's instructions. Using the above data, results for all the additional known common variants (MAF $>0.02$ in Europeans) on the January 2012 release of the 1000 Genomes Project were imputed using IMPUTE version 2.0. Quality control and imputation steps were carried out separately in the different consortia, leading to slight differences in the numbers of SNPs with available data. In addition to the 902 successfully genotyped SNPs, genotypes at 2,972 SNPs were imputed in BCAC and 2,907 SNPs were imputed in CIMBA (imputation $r^{2}$ score $>0.3$ in each case). In total, 3,872 genotyped or imputed SNPs were available for the combined BCAC ER ${ }^{-}$and CIMBA $B R C A 1$ mutation carrier meta-analysis

Statistical analysis. Case-control analysis, logistic regression and retrospective cohort analyses. For the case-control analysis in BCAC, per-allele odds ratios and standard errors were estimated for each SNP using logistic regression, separately for subjects of European and Asian ancestry and for each tested phenotype. Principal components were included as covariates as previously described $^{21}$. The statistical significance of each SNP was derived using a Wald test. To evaluate evidence for multiple association signals, we performed conditional analyses in which the association for each SNP was reevaluated after including other associated SNPs in the model. SNPs with a $P$ value $<1 \times 10^{-4}$ and MAF $>2 \%$ in the single-SNP analysis were included in this analysis ${ }^{21}$. Haplotype-specific odds ratios and confidence limits were estimated using haplo.stats ${ }^{22}$.

Associations between genotypes and breast cancer risk in BRCA 1 mutation carriers in CIMBA were evaluated using a per-allele trend test with 1 degree of freedom $\left(P_{\text {trend }}\right)$, based on modeling the retrospective likelihood of the observed genotypes conditional on breast cancer phenotypes ${ }^{41}$. To allow for non-independence among related individuals, an adjusted test statistic was used that took into account the correlation in genotypes ${ }^{21}$. Per-allele hazard ratio estimates were obtained by maximizing the retrospective likelihood. All analyses were stratified by country of residence.

Conditional analyses were performed to identify SNPs independently associated with each phenotype. To identify the most parsimonious model, all SNPs with a marginal $P$ value $<1 \times 10^{-4}$ were included in forward selection regression analyses with a threshold for inclusion of $P<1 \times 10^{-4}$ and including terms for principal components and study. Similarly, forward selection Cox regression analysis was performed for BRCA1 mutation carriers, stratified by country of residence, using the same $P$-value thresholds. This approach provides valid significance tests of the associations, although the estimates quantifying the association can be biased ${ }^{41,42}$. Parameter estimates for the most parsimonious model were obtained using the retrospective likelihood approach.

Within MODE, mammographic dense area, non-dense area and percent dense area were each square-root transformed to fit a normal distribution. For the ten MODE and BCAC studies, a linear regression assuming a multiplicative per-allele model adjusting for study, age at mammogram, BMI, menopausal status (pre or post) and the first six principal components was carried out for each trait and for each SNP. The MMHS participants were analyzed separately in the same way but without the principal-components covariates, and the results were combined with those from BCAC using a standard inverse variance-weighted fixed-effects meta-analysis.

Expression analysis. eQTL analyses were conducted in 57 normal breast samples from the GTEx Project ${ }^{43}$ and 135 adjacent normal breast samples from women of European origin in the METABRIC study ${ }^{44}$. For the METABRIC analyses, matched gene expression (Illumina HT-12 v3 microarray) and germline SNP data from either genotyping (Affymetrix SNP 6.0) or imputation (1000 Genomes Project, March 2012 data using IMPUTE version 2.0) were used. Correlations between the five signal-representative SNPs and expression levels of nearby genes (500 kb upstream and downstream of the SNPs) were assessed using a linear regression model in which an additive effect on expression level was assumed for each copy of the rare allele. Calculations were carried out using the eMAP library in R.

Allele-specific expression analysis. ASE analysis has been described previously ${ }^{11}$. Three SNPs for signal 1, two SNPs for signal 3 and a proxy SNP for signal $2\left(r^{2}=0.85\right)$ were on Affymetrix SNP Array 6.0. TCGA genotype calls and corresponding confidence scores were retrieved using level 2 TCGA SNP array Birdseed data downloaded from the TCGA portal. Genotyping data with a confidence score of 0.1 were excluded. We selected 742 breast cancer samples with European ancestry. The corresponding RNA-seq BAM files and metadata are available from the Cancer Genomics Hub (CGHub). Marker SNPs, the exonic SNPs of the target genes, were extracted from dbSNP human Build 142 (collectively 800 SNPs for ESR1, RMND1, ARMT1 and CCDC170), and RNA-seq read counts on SNP sites for reference and alternative alleles were computed. Homozygote marker SNPs and those with low coverage (less than $15 \times$ ) were excluded. Major allele fraction $(\mu)$ representing allelic imbalance for each marker SNP was computed, and an average of allelic imbalances for each gene was calculated for individual tumor samples. Marker SNPs with extreme $\mu$ values $(\mu>0.75)$ were not included in the analysis. Level 3 SNP array data were downloaded from the TCGA portal, and GISTIC version 2.0.16 
was used to identify copy number variations (CNVs) for each sample. Samples with low or high CNV levels, as presented in the gene-based GISTIC module report, were excluded from the analysis of the corresponding gene. For each risk SNP, allelic imbalance for the target transcripts was compared between heterozygote $(\mathrm{AB})$ and homozygote $(\mathrm{AA}$ and $\mathrm{BB})$ samples. For a given risk SNP and target gene, we used Levene's test, a more robust test than the $F$ test, for equality of variances when the risk SNP was not in linkage disequilibrium with any of the marker SNPs on that gene $\left(r^{2}<0.5\right)$. Otherwise, a two-tailed $t$ test was used for equality of means ${ }^{45}$.

Estrogen receptor protein expression. Normal breast samples derived from 150 postmenopausal donors (non-Hispanic, mean age of 62 years) and identified through the Susan G. Komen for the Cure Tissue Bank at the Indiana University Simon Cancer Center were used in this study ${ }^{46}$. DNA was extracted from blood cells at the Indiana CTSI Specimen Storage Facility using an AutogenFlex Star instrument (Autogen) and the FlexiGene AGF3000 blood kit for DNA extractions (Qiagen). SNP analysis was performed with $1 \mathrm{ng}$ of DNA using TaqMan genotyping assays for rs2046210 (C_12034236_10), rs3757322 (C_27475059_10), rs9397437 (C_11556300_10), rs851984 (C_2496819_10), rs9918437 (C_29496189_10) and rs2747652 (C_2823750_10) from Life Technologies, following the manufacturer's protocol. ER protein abundance was measured by immunohistochemical semiquantitation using an antibody to $\mathrm{ER} \alpha$ (clone 6F11; 1:40 dilution; Leica Microsystems) and quantified with (i) an $\mathrm{H}$ score consisting of the sum of the percent of tumor cells staining, multiplied by an ordinal value corresponding to the intensity level (0, none; 1, weak; 2, moderate; 3, strong; Supplementary Fig. 2), and (ii) the percentage of positive cells. Correlations between the $\mathrm{H}$ scores and ER immunohistochemistry values were calculated using Spearman's rank correlation analysis. All $P$ values reported are two-sided, and values $<0.05$ were considered statistically significant.

Cell lines. Breast cancer cell lines MCF-7 (ER+; American Type Culture Collection (ATCC) HTB22), T-47D (ER ${ }^{+}$; ATCC HTB133) and BT-474 (ER+; ATCC HTB20) were grown in RPMI medium with 10\% FCS and antibiotics. Normal breast epithelial cell lines MCF10A (ATCC CRL 10317) and Bre80 (provided as a gift from R. Reddel, Children's Medical Research Institute, Sydney) were grown in DMEM/F12 medium with 5\% horse serum (HS), $10 \mu \mathrm{g} / \mathrm{ml}$ insulin, $0.5 \mu \mathrm{g} / \mathrm{ml}$ hydrocortisone, $20 \mathrm{ng} / \mathrm{ml}$ epidermal growth factor, $100 \mathrm{ng} / \mathrm{ml}$ cholera toxin and antibiotics. Cell lines were maintained under standard conditions, routinely tested for mycoplasma and short tandem repeat (STR) profiled.

Chromatin conformation capture. $3 \mathrm{C}$ libraries were generated using EcoRI, HindIII or BglII as described previously ${ }^{15}$. 3C interactions were quantified by RT-PCR (qPCR) using primers designed within restriction fragments (Supplementary Table 15). qPCR was performed on a RotorGene 6000 instrument using MyTaq HS DNA polymerase (Bioline) with the addition of $5 \mathrm{mM}$ Syto9, an annealing temperature of $66^{\circ} \mathrm{C}$ and an extension time of $30 \mathrm{s.} 3 \mathrm{C}$ analyses were performed in three independent $3 \mathrm{C}$ libraries from each cell line, with each experiment quantified in duplicate. BAC clones (RP11-108N8, RP11-713G5, RP11-450E24 and RP11-55K19) covering the 6q25 region were used to create artificial libraries of ligation products to normalize for PCR efficiency. Data were normalized to the signal from the BAC clone library and, between cell lines, by reference to a region within GAPDH. All qPCR products were electrophoresed on $2 \%$ agarose gels, gel purified and sequenced to verify the $3 \mathrm{C}$ product.

Electromobility shift assays. Gel shift assays were performed with $\mathrm{ER}^{+} \mathrm{MCF}-7$ or $\mathrm{ER}^{-}$Bre-80 nuclear lysates and biotinylated oligonucleotide duplexes (Supplementary Table 16). Nuclear lysates were prepared using NE-PER nuclear and cytoplasmic extraction reagents (Thermo Fisher Scientific) according to the manufacturer's instructions. Total protein concentrations in nuclear lysates were determined by Bradford's method. Duplexes were prepared by combining sense and antisense oligonucleotides in NEBuffer2 (New England BioLabs) and heat annealing at $80{ }^{\circ} \mathrm{C}$ for $10 \mathrm{~min}$ followed by slow cooling to $25^{\circ} \mathrm{C}$ for $1 \mathrm{~h}$. Binding reactions were performed in binding buffer (10\% glycerol, 20 mM HEPES (pH 7.4), 1 mM DTT, protease inhibitor cocktail
(Roche), $0.75 \mu \mathrm{g}$ poly(dI:dC) (Sigma-Aldrich)) with $7.5 \mu \mathrm{g}$ of nuclear lysate. For competition assays, binding reactions were preincubated with 1 pmol of competitor duplex (Supplementary Table 17) at $25^{\circ} \mathrm{C}$ for $10 \mathrm{~min}$ before the addition of $10 \mathrm{fmol}$ of biotinylated oligonucleotide duplex and a further incubation at $25^{\circ} \mathrm{C}$ for $15 \mathrm{~min}$. Reactions were separated on 10\% Tris-borate-EDTA (TBE) polyacrylamide gels (Bio-Rad) in TBE buffer at $160 \mathrm{~V}$ for $40 \mathrm{~min}$. Duplex-bound complexes were transferred onto Zeta-Probe positively charged nylon membranes (Bio-Rad) by semidry transfer at $25 \mathrm{~V}$ for $20 \mathrm{~min}$ and then cross-linked onto the membranes under 254-nm ultraviolet light for $10 \mathrm{~min}$. Membranes were processed with the LightShift Chemiluminescent EMSA kit (Thermo Fisher Scientific) according to the manufacturer's instructions. Chemiluminescent signals were visualized with the C-DiGit blot scanner (LI-COR).

Plasmid construction and reporter assays. Promoter-driven luciferase reporter constructs were generated by the insertion of PCR-amplified fragments containing ESR1, ARMT1, RMND1 and CCDC170 promoters into the KpnI and MluI sites of pGL3-Basic. To assist in cloning, AgeI and SbfI sites were inserted into the BamHI and SalI sites downstream of the luciferase gene. A 1,496-bp signal 1 PRE fragment, a 997-bp signal 2 PRE fragment, a 1,566bp signal 3 PRE fragment, a 1,463-bp signal 4 PRE fragment and a 1,349-bp signal 5 PRE fragment were generated by PCR or gBlocks (Integrated DNA Technologies) and cloned into the AgeI and SbfI sites of the modified pGL3Promoter constructs. The minor alleles of individual SNPs were introduced into the PRE sequences by overlap extension PCR or gBlocks. Sequencing of all constructs confirmed variant incorporation (AGRF). ER ${ }^{+}$MCF-7 and BT-474 or ER ${ }^{-}$Bre- 80 cells were transfected with equimolar amounts of luciferase reporter plasmids and $50 \mathrm{ng}$ of pRL-TK transfection control plasmid with Lipofectamine 3000. The total amount of transfected DNA was kept constant at $600 \mathrm{ng}$ for each construct by the addition of pUC19 as a carrier plasmid. Luciferase activity was measured $24 \mathrm{~h}$ after transfection by the DualGlo Luciferase Assay System. To correct for any differences in transfection efficiency or cell lysate preparation, firefly luciferase activity was normalized to Renilla luciferase activity, and the activity of each construct was measured relative to the promoter-only construct, which had a defined activity of 1 . Statistical significance was tested by log transforming the data and performing two-way ANOVA followed by Dunnett's multiple-comparisons test in GraphPad Prism.

Chromatin immunoprecipitation. $\mathrm{ER}^{+} \mathrm{MCF}-7$ and BT-474 breast cancer cells were cross-linked with $1 \%$ formaldehyde at $37^{\circ} \mathrm{C}$ for $10 \mathrm{~min}$, rinsed once with ice-cold PBS containing 5\% BSA and once with PBS, and collected in PBS containing $1 \times$ protease inhibitor cocktail (Roche). The cells were centrifuged for $2 \mathrm{~min}$ at $900 \mathrm{~g}$. Cell pellets were resuspended in 0.35 $\mathrm{ml}$ of lysis buffer (1\% SDS, $10 \mathrm{mM}$ EDTA, $50 \mathrm{mM}$ Tris- $\mathrm{HCl}, \mathrm{pH} 8.1,1 \times$ protease inhibitor cocktail) and sonicated three times for $15 \mathrm{~s}$ each with a $70 \%$ duty cycle (Branson SLPt) followed by centrifugation at 15,000 $\mathrm{g}$ for $15 \mathrm{~min}$. Supernatants were collected and diluted in dilution buffer (1\% Triton X-100, $2 \mathrm{mM}$ EDTA, $150 \mathrm{mM} \mathrm{NaCl}, 20 \mathrm{mM}$ Tris-HCl, $\mathrm{pH}$ 8.1). Two micrograms of antibody was prebound for $6 \mathrm{~h}$ to Protein G Dynabeads (Life Technologies) and then added to the diluted chromatin for overnight immunoprecipitation. The magnetic bead-chromatin complexes were collected and washed six times in RIPA buffer (50 mM HEPES, pH 7.6, 1 mM EDTA, 0.7\% sodium deoxycholate, $1 \% \mathrm{NP}-40,0.5 \mathrm{M} \mathrm{LiCl}$ ) and then twice with TE buffer. To reverse the cross-linking, the magnetic bead complexes were incubated overnight at $65^{\circ} \mathrm{C}$ in elution buffer ( $\left.1 \% \mathrm{SDS}, 0.1 \mathrm{M} \mathrm{NaHCO}_{3}\right)$. DNA fragments were purified using a QIAquick Spin kit (Qiagen). For qPCR, $2.0 \mu \mathrm{l}$ from a 100- $\mu \mathrm{l}$ immunoprecipitated chromatin extraction was subjected to 40 cycles of amplification. All PCR products were sequenced by Sanger sequencing (AGRF). The antibodies used were to CTCF (C-20; sc-15914) and GATA3 (HG3-31; sc268) or control IgG (sc-2027) (all from Santa Cruz Biotechnology). ChIP primers are listed in Supplementary Table 18.

37. Chenevix-Trench, G. et al. An international initiative to identify genetic modifiers of cancer risk in BRCA1 and BRCA2 mutation carriers: the Consortium of Investigators of Modifiers of BRCA1 and BRCA2 (CIMBA). Breast Cancer Res. 9, 104 (2007).

38. Couch, F.J. et al. Genome-wide association study in BRCA1 mutation carriers identifies novel loci associated with breast and ovarian cancer risk. PLoS Genet. 9, e1003212 (2013). 
39. Boyd, N.F. et al. Mammographic density and the risk and detection of breast cancer. N. Engl. J. Med. 356, 227-236 (2007).

40. Dunning, A.M. et al. Association of ESR1 gene tagging SNPs with breast cancer risk. Hum. Mol. Genet. 18, 1131-1139 (2009).

41. Barnes, D.R., Lee, A., Easton, D.F. \& Antoniou, A.C. Evaluation of association methods for analysing modifiers of disease risk in carriers of high-risk mutations. Genet. Epidemiol. 36, 274-291 (2012).

42. Antoniou, A.C. et al. A weighted cohort approach for analysing factors modifying disease risks in carriers of high-risk susceptibility genes. Genet. Epidemiol. 29, 1-11 (2005).
43. GTEx Consortium. The Genotype-Tissue Expression (GTEx) project. Nat. Genet. 45 580-585 (2013).

44. Curtis, C. et al. The genomic and transcriptomic architecture of 2,000 breast tumours reveals novel subgroups. Nature 486, 346-352 (2012).

45. Xiao, R. \& Scott, L.J. Detection of cis-acting regulatory SNPs using allelic expression data. Genet. Epidemiol. 35, 515-525 (2011).

46. Sherman, M.E. et al. The Susan G. Komen for the Cure Tissue Bank at the IU Simon Cancer Center: a unique resource for defining the "molecular histology" of the breast. Cancer Prev. Res. (Phila.) 5, 528-535 (2012). 